\title{
THE EVOLUTION OF STARS OF MEDIUM MASS
}

\author{
Cesare Chiosi \\ Department of Astronomy \\ University of Padova \\ Vicolo dell'osservatorio 5 \\ 35122 Padova, Italy
}

Abstract. The main properties of the evolution of low, intermediate, and high mass stars are reviewed focusing on a few issues tightly related to the interpretation of Pop I Cepheid stars. After a summary discussion of the physical mechanism responsible for the Cepheid pulsation, the classical results of stellar evolution theory for the main evolutionary phases (main sequence, core He-burning, and later) all over the HR diagram are presented, putting into evidence the various points of disagreement with current observational data. We then review the models incorporating the effect of convective overshoot, and present in some detail a study on the rich, young cluster NGC 1866 in the Large Magellanic Cloud, in which they are compared with the observational data. Arguments are given to favour the adoption of models with convective overshoot instead of the classical ones. The topics of the mass discrepancy and frequency-period distribution of Cepheid stars are then discussed in the light of models with convective overshoot, showing how they can better account for the observational data. Finally, we address the question of the enhancement in the radiative opacity of heavy elements in the middle temperature region (approximately where the ultimate ionization of the CNO group of elements occurs), which has been suggested to explain the mass anomalies and period ratios encountered in double-mode and bump Cepheids. Evolutionary models for intermediate mass stars incorporating the above modification in the radiative opacity are then presented and discussed in some detail.

\section{INTRODUCTION}

Becker (1985), in his recent review on the evolution of intermediate mass stars in relation to Cepheids, separates these stars in two loose groups according to their metallicity: the Pop I Cepheids when $Z>0.005$, and Pop II Cepheids when $Z<0.005$. Galactic Pop I Cepheids have periods in the range 1-80 days, whereas those of the Large Magellanic Cloud may pulsate with periods up to 200 days. Galactic Cepheids are concentrated toward the galactic plane, and possess effective temperatures from 6600 to $5500 \mathrm{~K}$ (spectral type $\mathrm{F} 5$ to $\mathrm{G} 5$ ) and visual absolute magnitudes from -1 to -6 . These pulsators obey a well defined period-luminosity relationship, and from an evolutionary point of view are commonly thought to arise as the result of normal post main 
sequence evolution of intermediate to high mass stars ( 3 to $10 \mathrm{M}_{\odot}$ ) in core He-burning stages. With the possible exception of Pop II stars of relatively high mass $\left(M>2 M_{\odot}\right.$ ) that may occur in galaxies like LMC and SMC, and which would behave similarly to classical Pop I Cepheids, Pop II Cepheids are a separate class of stars which lie in the HR diagram between RR Lyrae, RV Tauri and LPV stars, obey a different periodluminosity relationship, are broken into three main groups (BL Her stars, $W$ Vir stars, and Anomalous Cepheids), and are generally associated to low mass stars undergoing post core He-burning evolution, although other explanations are possible for the last group.

\section{THE CLASSICAL SCENARIO FOR POPULATION I CEPHEIDS}

In the following, we will limit ourselves only to the Pop I Cepheids, paying major attention to those aspects tightly related to the evolutionary behaviour of intermediate and high mass stars.

There are two main facts that concur to determine the Cepheid star phenomenon: 1) the instability strip itself (location in the HR diagram, slope, width, etc.); 2) the crossing of the instability strip by a star in the course of its evolution.

In fact, whenever a star crosses the instability strip, it becomes pulsationally unstable according to the well known mechanisms, even if differences, arising from the particular evolutionary stage during which the crossing occurs, are likely to be expected.

\subsection{The Cepheid instability strip}

Although the physical mechanism responsible for the pulsation of Cepheid stars has been well known for a long time, so that a lengthy discussion is superfluous (see also the recent review by Cox 1985), nonetheless a short summary of the basic ideas may be useful. Given that nuclear reactions play a totally negligible role in the phenomenon of pulsation in most types of variable stars, since pulsations in stellar interiors, where nuclear reactions occur, are of very small amplitude, the source of instability has been identified with the envelope ionization mechanism. The ability of the envelope ionization to drive pulsations resides in the fact that the ionization of abundant elements results in a modulation of the flux variations, and these modulations are such that the regions absorb heat when they are most compressed and lose heat when most expanded. As a consequence of this, maximum pressure in these layers will come after maximum density, thus favouring instability. Finally, the ionization region is located in the external layers of a star where the pulsation amplitudes happen to be high. In contrast, the innermost regions oscillate at small amplitudes and show the opposite tendency, thus damping all incipient pulsations. The effects of modulations of the flux can be understood by means of the work integral, written in the approximate form (see J. P. Cox 1980 for details)

$$
W=-L_{T} \int_{0}^{M}\left(\Gamma_{3}-1\right)(\partial \rho / \rho) d / d m(\partial L / L) d m
$$


where all variations denote the space parts of the corresponding variables, nuclear energy sources are neglected, $I$ is the interior luminosity at radius $r,(\partial \rho / \rho)$ is assumed real and positive (regions are compressed at minimum stellar radius), and finally the equilibrium value of $\mathrm{L}$ is assumed constant with the mass and equal to the total luminosity $L_{T}$. Negative and positive values of $W$ indicate stability and instability, respectively. Assuming that the energy is carried only by radiation (a reasonable approximation in most cases), the luminosity variations are given by

$$
(\partial L / L)=4(\partial r / r)-(\partial k / k)+4(\partial T / T)+\left(d r_{0} / d\left[\ln T_{0}\right]\right) d / d r_{0}(\partial T / T),
$$

where the quantities in brackets have their usual meaning and the subscript o refers to the equilibrium configuration. Using the quasiadiabatic approximation,

$$
(O T / T)=\left(\Gamma_{3}-1\right)(O \rho / \rho),
$$

assuming a Kramers-like law for the opacity $k=\kappa_{0} \rho^{n} T^{-s}$, and neglecting the last term in equation (2) we get,

$$
(\partial L / L)_{a}=4(\partial r / r)+\left[(s+4)\left(\Gamma_{3}-1\right)-n\right](\partial \rho / \rho)
$$

together with

$$
(\partial L / L)=4(\partial r / r)-n(\partial \rho / \rho)(s+4)(\partial T / T) .
$$

In most physical situations, since $d(\partial \mathrm{L} / \mathrm{L}) / d m$ turns out to be positive, $W$ is negative, and stability is established. This can be easily understood as follows: at maximum compression, the luminosity variations increase outward, hence more heat flows out from each elementary shell than flowing in from inside and the pulsations are damped. This is a result of the opacity variation, which usually decreases upon compression (see the temperature, density dependence of the Kramers law). The important effect of the ionization of abundant elements like $\mathrm{H}$, He, $\mathrm{C}$, and $\mathrm{O}$ is that $\left(\Gamma_{3}-1\right)$ becomes very small, since most of the work of adiabatic compression goes into ionization rather than into kinetic energy of thermal motion, so that temperature remains about constant upon compression. This can be easily understood considering that ionization represents additional internal degrees of freedom. As a consequence of it, the adiabatic luminosity variations exhibit a marked dip in the ionization zone. The strong outward decrease of $(\partial \mathrm{L} / \mathrm{L})_{a}$ in the inner part of the ionization zone indicates absorption of energy at maximum compression and acts as the driving source of destabilization (see equation (1)). The diminution of $\left(\Gamma_{3}-1\right)$ and $(\delta \mathrm{L} / \mathrm{L})_{\mathrm{a}}$ results in part from the smaller temperature variation in occurrence of ionization. This direct effect of the temperature variations on the luminosity variations is referred to as the $\mathrm{r}$-mechanism. If the opacity obeys a Kramer-like relation, it follows that the small values of $(0 \mathrm{~T} / \mathrm{T})$ in regions of small $\left(\Gamma_{3}-1\right)$ may cause $\mathrm{k}$ to increase upon compression, whereas normally the opposite occurs. This local increase in opacity contributes still further to the driving 
(instability). This constitutes the so-called $k$-mechanism.

Furthermore, upon compression, negative $(0 r / r)$, the total radiating area is reduced, so that more energy is trapped into the region, thus contributing to destabilize it still further. This is referred to as the $r$-mechanism [first term in equations (4) and/or (5)]. Finally, if the exponent $s$ is large and negative (as may be the case in the $H$ ionization zone), there may be a damming up of radiation upon compression, hence driving, even if $\Gamma_{3}-1$ has the normal value. This fact has been found important in stars like $\partial$ Scuti and $\beta$ Cephei (Stellingwerf 1978, 1979) and it is referred to as the "bump mechanism". Arguments similar to those developed above lead to the conclusion that $(\mathrm{oL} / \mathrm{L})_{a}$ must increase rapidly outward in the outer layers of the ionization zone, thus giving the opposite effect. In general, the strong driving of the innermost portions is largely cancelled by the opposite trend in the outermost portions. However, because of nonadiabatic effects, $(\partial \mathrm{L} / \mathrm{L})$ and $(\partial \mathrm{L} / \mathrm{L})$ a may be quite different in the very outer layers of a star. To understand the role of the nonadiabatic behaviour of the layers far out in a star, it is convenient to distinguish three separate regions. The first is the inner region in which oscillations are usually nearly adiabatic and any driving is almost exactly cancelled by an equal amount of damping, so that there is no net contribution. The second is the outermost region, which may contain a large fraction of the radius and in which ionization and highly nonadiabatic effects may occur, but whose mass is so small that the perturbations of the radiative flux are effectively "frozen in" so that, owing to the absence of modulation, there is no net driving. The third region, called the transition region, $T R$, is located in between the first and the second one, and can be roughly defined by the condition that the total internal energy of the layers above it equals the energy radiated by the star in one pulsation period,

$$
\left(c_{v} T^{*} \Delta M^{*}\right)=L P \text {, }
$$

where $c_{v}$ is the specific heat at constant volume, $T^{*}$ is the mean temperature of the $T R, \Delta M^{*}$ is the mass above it, $L$ is the luminosity, and $P$ the period. The location of the TR is mostly regulated by the equilibrium radius $R$ of the star $\left(T \propto R^{-0.5}\right.$ ) and it moves outward in mass as $R$ increases. The main effects of nonadiabaticity in the outer layers are to decouple (OL/L) from (oL/L)a in the regions exterior to $T R$. Density and temperature variations are such that (OL/L) is kept about constant. If $R$ is so small that the ionization zones lie above the $T R,(\partial L / L)$ is constant in the region in spite of the pronounced dip in $(\partial \mathrm{L} / \mathrm{L})_{a}$. Therefore $(\partial \mathrm{L} / \mathrm{L})$ is positive and increases outward until the TR is reached and then remains constant. Therefore only damping exists in the outer layers and the star is stable. At somewhat larger radii, the TR moves inward and eventually becomes nearly equal to the characteristic temperature of ionization of elements like He. In this case the ionization region and the TR almost coincide. The inner layers of the ionization zone essentially lie in the adiabatic interior, where $(\partial \mathrm{L} / \mathrm{L})=(\mathrm{L} / \mathrm{L}) \mathrm{a}$ and where the outward decrease in $\left(\Gamma_{3}-1\right)$ induces a strong decrease both in $(\delta \mathrm{L} / \mathrm{L})_{a}$ and $(\mathrm{LL} / \mathrm{L})$. The outer layers of the ionization region lie essentially in the nonadiabatic domain, where 
$(\partial \mathrm{L} / \mathrm{L})$ has become frozen in at the small value it had at the dip. In this case the strong damping in the outer layers is eliminated by nonadiabatic effects, and the strong driving due to the rapid decrease of $(\partial \mathrm{L} / \mathrm{L})$ in the inner layers is now effective and the star is unstable. It can be easily seen that condition ( 6 ) defining the TR implies that the instability region is sharply bounded on the high effective temperature side, and that this boundary is nearly vertical on the HR diagram. The large body of literature on the Cepheid star instability strip has clarified that the $\mathrm{He}^{+}$envelope ionization is the driving agent of their pulsation and the detailed calculations confirm the above oversimplified picture (see Cox 1985 for updated references). The envelope ionization mechanism, which so successfully accounts for the blue side of the instability strip, does not account, on the basis of pure radiative transfer, for the return to stability indicated by the red side of the strip. However, as the radius of the star increases, convection sets in in the outer layers and simple minded arguments permit us to see that convection in the envelope actually quenches pulsations. In fact, convective transfer is most efficient when the star is most compressed. Near maximum compression, the rate of energy losses is most effective, yielding an ideal situation for damping and hence stability (Deupree 1977). Unfortunately, the modulation of the flux upon the occurrence of convection is much more complicated than in the radiative case, so that the exact determination of the red boundary of the instability strip is uncertain. This implies that the width of the instability strip is still a matter of debate. Along the blue edge of the instability strip we may express condition (6) in an alternative form

$$
L=Q_{i}^{\alpha} \quad Y^{\beta} Z^{Y} M^{\partial} \quad T_{\text {eff }}^{\partial}
$$

where $l_{i}$ is the pulsational constant for the mode under consideration, $Y$ and $\mathrm{Z}$ are, respectively, the helium and heavy element abundances, and $a_{\text {, }}$ $\beta, \quad y$, and $\delta$ are appropriate exponents to be determined by detailed pulsation calculations (see Iben \&uggle 1972a,b, 1975). Relation (7) has a number of important applications. For instance, it embodies the period luminosity relationship. If $\mathrm{T}_{\text {eff }}$ is expressed as a function of $L$, and $R$, and $R$ in turn is expressed as a function of $P, M$ and $Q_{i}$ are taken from the period-mean density relationship, then at given $Y$ and $Z$ relation (7) becomes a relation among $L, M$ and $P$ and, in turn, assuming a $L-M$ dependence, it yields $L=L(P)$ (see also J.P. Cox 1985). The period is known to increase with the luminosity. Equation (7) is also useful to estimate how the blue edge of the instability strip is affected by variations in the chemical composition. At fixed $L$, an increase in $\mathrm{Y}$ moves the blue edge to higher $\mathrm{T}_{\text {eff }}$, whereas an increase in $\mathrm{Z}$ moves the blue edge to lower $\mathrm{T}_{\text {eff }}$. This arises primarily through the opacity; an increase in $Y$ implies a decrease in the hydrogen content (normally the main source of opacity in the envelope). Since the material in the envelope is more transparent, the $\mathrm{He}^{+}$ionization temperature is reached at higher pressure or equivalently for larger values of $\Delta \mathrm{M}^{*}$. Therefore $\mathrm{T}_{\text {eff }}$ must increase in order to satisfy equation (6). Similarly for variations in $z$. Furthermore, the blue edge of higher mode pulsations (smaller $Q_{i}$ ) should in general lie at 
higher $T_{\text {eff }}$ than a lower mode blue edge. This prediction is however not always confirmed by detailed calculations (Iben \& Tuggle 1972a,b) since equation (6) is only a necessary, not a sufficient, condition for instability.

\subsection{The classical picture for the evolution of intermediate and high mass stars \\ In the following, we will consider only those stars which,} by virtue of the relatively high initial mass and He core mass, avoid the core He-flash and ignite helium in non-degenerate conditions. As is well known the minimum core mass for this to occur is about 0.45 to $0.55 \mathrm{M}_{\mathrm{e}}$, with which an initial mass (otherwise known as $\mathrm{M}_{\mathrm{HeF}}$ ) of about 2.2 to $1.8 \mathrm{M}_{\odot}$ is associated, respectively, according to the initial chemical composition. Stars lighter than $\mathrm{M}_{\mathrm{HeF}}$ are not of interest here. They in fact either never cross the instability strip (or do it only once on a very short time scale) or, crossing it during the core He-burning phase in the horizontal branch (HB), give rise to the RR Lyrae stars, pulsators whose instability mechanism is similar to that of Cepheids, but whose periods are of the order of a few hours. In turn we distinguish the intermediate mass stars from the massive ones by looking at the stage of carbon ignition in the core. By intermediate mass we mean those stars which, following core He-exhaustion, develop an highly degenerate carbon-oxygen $(\mathrm{C}-\mathrm{O})$ core, and as asymptotic giant branch (AGB) stars experience helium shell flashes or thermal pulses. The AGB phase is terminated either by envelope ejection and formation of a white dwarf $\left(\mathrm{M}_{\mathrm{HeF}}<\mathrm{M}_{\mathrm{i}}<\mathrm{M}_{\mathrm{W}}\right)$ or carbon ignition and deflagration in a highly degenerate core once it has grown to the Chandrasekhar limit of $1.4 \mathrm{M}_{\odot}$. The limit mass $\mathrm{M}_{W}$ is regulated by the efficiency of mass loss by stellar wind during the red giant (RGB) and AGB phases (see Iben \& Renzini 1983). This point will be discusssed in more detail below. The minimum mass of the $\mathrm{C}-\mathrm{O}$ core, below which carbon ignition in non-degenerate condition fails and the above scheme holds is $1.06 \mathrm{M}_{\odot}$ to which an initial mass from 7 to $9 \mathrm{M}_{\odot}$ corresponds, depending on the chemical composition. This particular value of the initial mass is known as $M_{u p}$. Finally, massive stars are those that ignite carbon non-violently and through a series of nuclear burnings proceed either to the construction of an iron core and subsequent photodissociation instability with core collapse and supernova explosion $\left(\mathrm{M}_{\mathrm{i}}>\mathrm{M}_{\text {mas }}\right)$, or following a more complicated scheme undergo core collapse and supernova explosion ( $\mathrm{M}_{\text {up }}<\mathrm{M}_{i}<\mathrm{M}_{\text {mas }}$ ). $\mathrm{M}_{\text {mas }}$ is about $12 \mathrm{M}_{\odot}$. As a conclusion, the range of initial masses that matters in our discussion is confined between $\mathrm{M}_{\mathrm{HeF}}$ and an upper value of the mass, called $\mathrm{M}_{\text {Ceph }}$, located in the domain of massive stars (approximately $30 \mathrm{M}_{\odot}$ ), above which under the action of mass loss by stellar wind (see below) stars never get into the red supergiant area and, therefore, never intersect the instability strip.

i) Core H-burning. When, as a result of the pre-main sequence overall gravitational contraction, temperatures near the centre become sufficiently large that $\mathrm{H}$-burning reactions are initiated, the star settles on the main sequence, where it remains for most (up to 80\%) of 
its entire nuclear burning life. Conversion of hydrogen into helium in the core, causes the increase in the mean molecular weight together with core contraction and heating up determined by the release of gravitational energy. In the mass range we are interested in, the transfer of the liberated energy (nuclear and gravitational) is secured by convection, which, mixing the material over a finite fraction of the stellar mass, on one hand fuels the innermost regions of fresh hydrogen, thus determining the duration of the H-burning phase, on the other hand gives rise to the building up of a helium-rich core. The extension of the convective core is classically determined by the so-called Schwarzschild condition in a local, adiabatic treatment of convection. The mass fraction of the convective core and, in turn, the mass of the He-rich core at the end of the central H-burning, increase with the star mass, whereas the duration of the core H-burning decreases with it. During the core $\mathrm{H}$-burning phase the luminosity and radius of the star increase, whereas the effective temperature decreases. Core H-burning models are expected to describe a band in the HR diagram, whose width is essentially determined by the size of the convective core lamong the most massive stars also by the efficiency of mass loss by stellar wind and opacity in the CNO ionization region, see below), to which main sequence stars should correspond. The core H-burning phase of stars of any mass does not deserve a detailed discussion owing to the large body of literature existing on the subject. Suffice it to recall that massive stars may be affected by semiconvective instability and, more important, by mass loss by stellar wind, which in most massive stars may be efficient enough to deeply alter even the simple core H-burning phase (see Chiosi \& Maeder 1986).

Semiconvection (thereinafter the H-semiconvection) has long been the characterizing feature of the structure of massive stars evolved at constant mass. In brief, the existence of semiconvection is due to the fact that radiation pressure and electron scattering dominate pressure and opacity in massive stars. As a consequence of the high radiation pressure, the convective core tends to grow and to expand as evolution proceeds, giving rise to a chemical discontinuity at the border of the core, which due to the electron scattering opacity does not allow radiative equilibrium outside the border of the convective core to be established. The difficulty is removed assuming that partial mixing occurs to restore radiative equilibrium. The basic mechanism for the so-called semiconvective mixing is unknown however. Whether or not the matter in this region becomes fully convective during the overall contracting phase terminating the main sequence phase, depends on the choice for the stability criterion, over which there was considerable controversy (see Chiosi \& Maeder 1986 and references therein). The occurrence or non-occurrence of a fully convective shell influences where in the HR diagram core He-burning will take place. The advent of models with mass loss by stellar winds has put semiconvection aside till recently, when it has been opened up again in relation to the problem of the progenitor star of the supernova 1987A. 
To date the most salient signature of the evolution of massive stars is the occurrence of mass loss by stellar winds (see Chiosi \& Maeder 1986). The major differences with respect to the evolution at constant mass are that these stars evolve at lower luminosity, live longer and tend either to cover a wider range of effective temperatures or, on the contrary, to evolve near or to the left of the zero age main sequence as the rate of mass loss increases. Finally, semiconvection is strongly inhibited by mass loss by stellar wind. Fortunately, all this is seen to occur only in stars of very high mass $\left(\mathrm{M}_{1}\right.$ greater than about 30-40 $\left.\mathrm{M}_{0}\right)$, which, as a consequence of the high mass loss, never enter the red supergiant region and therefore are not relevant to our discussion of the Cepheid stars. Massive stars of relatively lower mass (say in the range 10 to $40 \mathrm{M}_{\odot}$ ) are only marginally affected by mass loss during the core H-burning phase, and they evolve similarly to constant mass stars. The major uncertainties are in the radiative opacities, and in the correct treatment of convection in central interiors, this latter giving rise to the well known phenomenon of convective overshoot (see below).

ii) Core He-burning. After hydrogen disappears at the centre, the H-exhausted core of the star contracts rapidly and, driven by the energy generated in a thin H-burning shell, the envelope expands. The star becomes a red giant and settles along the Hayashi line, where it undergoes the first dredge up episode, during which external convection may penetrate deep enough to pass the layer at which the chemical structure of the star has been previously altered by nuclear processing and/or internal mixing. In such a case changes in the surface chemical composition are expected. The H-exhausted core continues to grow in mass due to the outward migration of the H-burning shell, to contract and to heat up until He-burning temperatures are reached at the centre (this occurring, as already mentioned, well before electrons become degenerate there and while the star is at the tip of the Hayashi line). The question as to why stars become red giants has been debated for many years without receiving a satisfactory answer. The last studies of this topic are by Renzini (1984a), who identifies in a thermal instability in the envelope, primarily determined by the derivatives of the opacity in the middle temperature region (see also Iben \& Renzini 1984), the physical cause for the rapid expansion of the envelope to red giant dimensions. During the ensuing core He-burning phase, hydrogen continues to burn in a shell at about the same rate as it did during the main sequence phase. The rate at which helium is burnt in the convective core determines the rate at which the star evolves. Typically, the lifetime in the core He-burning stage is about 15 to $30 \%$ of the main sequence lifetime, being larger in models of smaller mass. Like the main sequence phase, at the end of the core He-burning, which turns helium into carbon, oxygen and traces of heavier species, a $\mathrm{C}-\mathrm{O}$ core is built up whose dimensions depend on the total mass of the star and once more the kind of physical model adopted to describe central convection and its efficiency. Since the mass of the convective core increases at increasing total mass, so does the mass of the C-O core. 
The core He-burning phase of intermediate mass stars, in particular of those toward the lower mass end, and of low mass stars (horizontal branch stages) is known to be affected by the same kind of convective instability (semiconvection) that was encountered by massive stars. The onset of this instability (hereafter the He-semiconvection) is due to the fact that as helium is turned into carbon and oxygen, the carbon-rich mixture inside the core is more opaque than the carbon-poor mixture outside the core. However, He-semiconvection, which requires a particular algorithm to properly fix the boundary of the convective core, alters the structure of intermediate mass stars only very marginally and can be ignored to any practical purpose. This is not the case for HB stars where the models are sensitive to the effects induced by semiconvection. Much more important, on the contrary, is the kind of convective instability met in the models at the very end of the core He-burning, when the central He content has dropped below a few per cent (Castellani et al. 1985). Although the physical nature of this instability, otherwise known as the breathing pulses of convection, is still uncertain, the net result of it is an oscillatory increase in the mass of the convective core, refueling the He-burning reactions, prolonging the core He-burning lifetime, and giving rise to a much larger C-O core. This phenomenon, although deeply affecting the ensuing AGB stages, has little relevance in the context of Cepheid stars.

Since all intermediate mass stars expand to red giant dimensions before igniting helium in the core, they cross the Cepheid instability strip at least once (first crossing). This is also true for most models of massive stars $\left(10<\mathrm{M}_{i}<40-50 \mathrm{M}_{\odot}\right)$ under favourable circumstances. However, since the above expansion takes place on the Kelvin-Helmholtz time scale $\left(10^{6}\right.$ to $10^{3}$ yr with increasing mass of the star), the first crossing has little relevance to the discussion of the properties of Cepheids, even though the possibility that some of these are in the first crossing cannot be excluded. Other possible crossings are discussed below.

iii) Evolution in the HR diagram during core He-burning. In intermediate mass stars, slow evolution during core He-burning takes place in two distinct regions, a first near the Hayashi line and a second at high effective temperatures. Stars of the first region are in early stages of core He-burning. Approximately, when the energy released by the burning core (increasing) equals the energy released by the H-burning shell (decreasing), a rapid contraction of the envelope readjusts the outer layers from convective to radiative and the star leaves the red region moving to high effective temperatures, where the major phase of core He-burning occurs. The location of these two regions and the lifetime spent in each region are function of the mass, chemical composition, nuclear reaction rates, the ${ }^{12} \mathrm{C}(\mathbf{a}, \mathrm{y}){ }^{16} \mathrm{O}$ in particular, extension of the convective core, opacity, mass loss along the red giant branch, and other both physical and computational details. For any choice of the composition, as the stellar mass decreases, the location of the blue region, otherwise known as the blue loop, moves toward the Hayashi line, and eventually merges with the red giant region. Thus for an assigned chemical composition, core He-burning 
occurs in bands, one roughly corresponding to the locus of the Hayashi line or red giant stars, and another that breaks off the red giant band at low luminosities and moves toward the blue with increasing luminosity (the so-called blue band). The mean slope of this is determined by the complicated interplay among the above physical factors and cannot be established a priori. Nonetheless, since the slope of the instability strip is opposite to that of the blue band defined by stars of a given composition in the core He-burning phase, the crossing of the two bands is likely to occur, unless unfavourable circumstances conspire to keep the whole core He-burning phase of stars of any mass in the red giant region, which is not the case. It goes without saying that even though a second and a third crossing are possible when the blue band develops, the most favourable case is given by those stars whose mass, chemical composition, etc. are such that the blue region coincides with or largely overlaps the instability strip. In such a case the instability strip is crossed on a nuclear time scale. The predicted locations in the HR diagram of stars of typical Pop I composition $(Y=0.28$ and $Z=$ $0.02)$ during the major nuclear-burning phases are shown in Figure 1. These models are calculated under standard assumptions for the treatment of the central convection, i.e. they ignore the effect of convective overshoot. The hatched regions visualize schematically where stars of all initial masses and ages spend most of their lifetime. As already recalled, stars more massive than $\mathrm{M}_{\mathrm{HeF}}$ have a core He-burning lifetime of about 15 to 25 per cent of the main sequence lifetime and share it among the two bands (red and blue). The location of the core He-burning phase of stars more massive than say $15 \mathrm{M}_{\odot}$ but lighter than about $50 \mathrm{M}_{\odot}$ is uncertain as the effect of mass loss by stellar wind during the whole stellar life is dominant. Nowadays calculations tend to favour models that spend the whole He-burning phase in the red supergiant region. Stars less massive than $\mathrm{M}_{\mathrm{HeF}}$, whose evolution is shortly summarized below, experience core He-flash which can be started only when the core mass has grown to a critical value (dependent on the composition) independent of the stellar mass. The growth of the core to the critical value occurs while matter there becomes highly electron degenerate and the star is ascending the RGB. During this phase external convection penetrates deeper and deeper, and the total mass of the star is decreased by about 20 per cent due to mass loss by stellar wind. The core He-flash occurs at the tip of the RGB at about the same luminosity for all stars (LogL/ $L_{\theta}=3.3$ ). The onset of the He-flash lifts degeneracy in the core and helium is thereafter burnt quiescently at higher temperatures and lower densities than at ignition. The constancy of the core mass implies that the lifetime of the following core He-burning phase (either as a HB or a red clump star) is independent of the mass. The typical duration of the phase is about $10^{8} \mathrm{yr}$. The location of the core He-burning stars, corresponds to the horizontal branch or red clump in the HR diagram depending on the mass and chemical composition. Under favourable circumstances (mostly the metal content) the HB can intersect the instability strip giving rise to the RR Lyrae pulsators. 
Figure 1. Theoretical HR diagram for classical models (no convective overshoot) evolved with mass loss by stellar wind in the domain of massive stars, and standard radiative opacities. The initial masses are given along the zero age main sequence. The hatched areas indicate the slow phases of nuclear burning. The initial chemical composition is $Y=0.28$ and $Z=0.02$. The location of the instability strip for the same chemical composition is indicated. The blue edge is for the fundamental mode, whereas the red edge is for the width $\Delta \log \mathrm{T}_{\text {eff }}=0.04 . \mathrm{L}_{\max }, \mathrm{L}_{0}$ and $\mathrm{L}_{\mathbf{1}}$ are three characteristic luminosities of the Cepheids (see text).

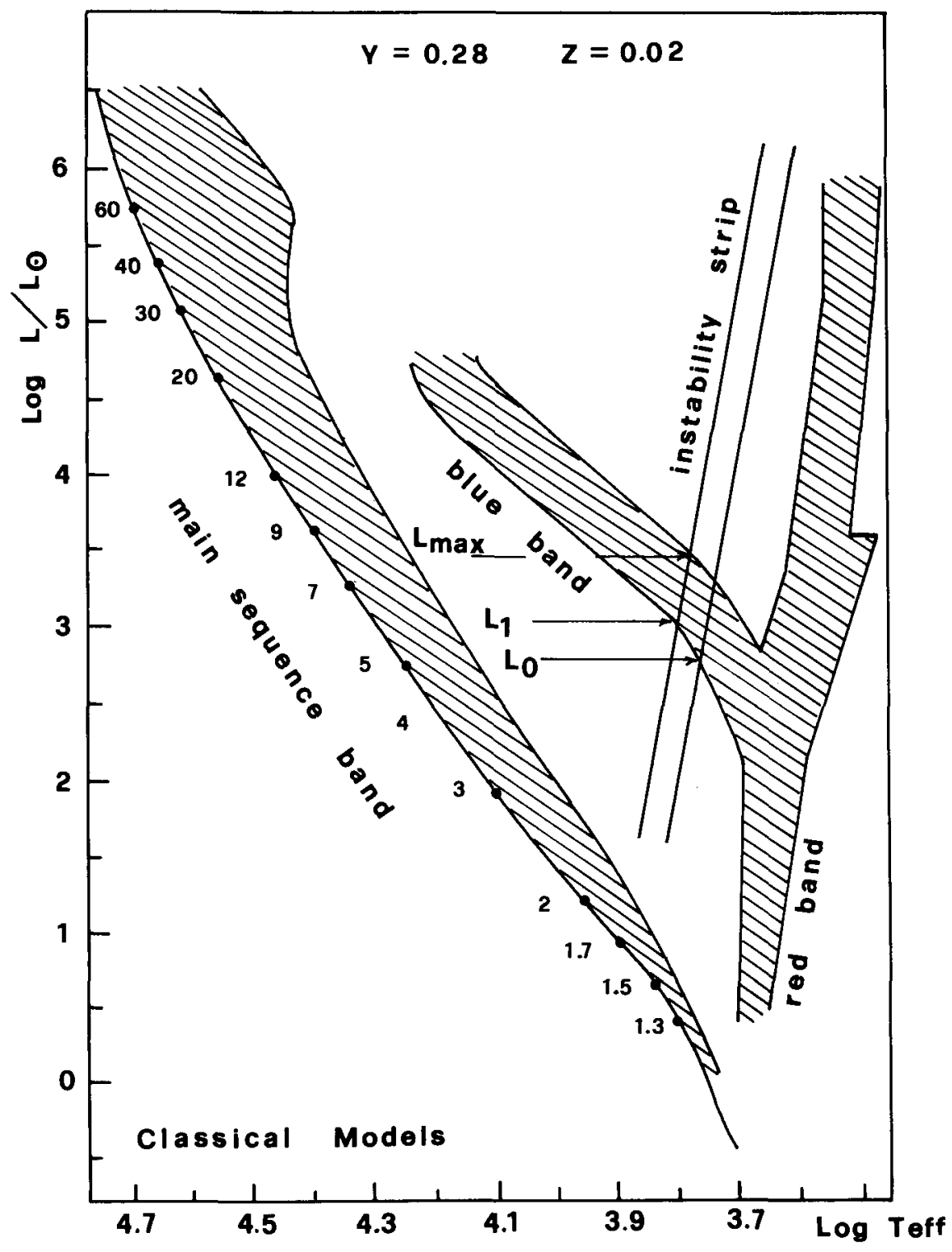


iv) Later evolutionary phases. All single stars less massive than $M_{u p}$ and heavier than say $0.7 \mathrm{M}_{\odot}$ become thermally pulsing AGB stars after exhausting helium in the core. AGB stars possess a C-O core, mostly oxygen with the new ${ }^{12} \mathrm{C}(a, y){ }^{16}$ o rate, within which electrons are higly degenerate, He- and H-burning shells separated by a thin layer of $\mathrm{H}$-exhausted matter, and an extended convective envelope. In consequence of the high thermal conductivity of degenerate electrons and loss of energy by an outflow of neutrinos produced by plasma and related processes, temperatures in the core remain below the treshold value for carbon ignition. Temperatures in the core are maintained at the high value $10^{8} \mathrm{~K}$ by the gravitational energy release from matter added to the core in virtue of the outward migration of the He- and H-burning shells. Sooner or later (for a more detailed discussion see Iben \& Renzini 1983, 1984), nuclear burning in the He shell becomes thermally unstable. In brief, the nuclear burning does not occur at a steady rate, but the two shells alternate with one another as the major source of energy. For 90 per cent of the time the He-burning shell is inactive and the H-burning shell is the major source of energy. However, as the mass of the He-rich zone below the H-burning shell increases, the density and temperature at the base of this zone increase until the rate of energy production by the $3 \mathbf{a}^{12} \mathrm{C}$ reaction gets higher than the rate at which energy can be carried out by radiative diffusion. A thermonuclear runaway occurs. A thin convective layer is generated on the top of the He-burning shell. At first the energy goes into raising the temperature of and expanding the matter in and near the burning zone, and the material is pushed away in both directions. Matter at the base of the $\mathrm{H}$-burning region is pushed out and cools to such low temperatures than the H-burning shell is temporarily extinguished. Eventually, matter at the He-burning region begins to cool as it overexpands and the rate of burning there drops dramatically. The convective layer disappears and a steady state is reached in which He-burning occurs quiescently at a slowly decreasing rate as the He-burning shell actually runs out of fuel. This quiescent phase lasts for about 10 per cent of the time elapsing betweeen successive outbursts. The material propelled out falls back and the H-burning shell eventually re-ignites. During this phase, by virtue of a complicated interplay among the outer convective envelope, the oscillatory nature of the shell burning, and the thin inner convective layer, material processed into the intershell region is brought into the outer convective envelope and exposed to the surface. The so-called third dredge up takes place. The pulsing regime of the AGB phase, which manifests itself in the HR diagram with continuous brightening of the star at about constant effective temperature, lasts until either the core has reached the critical value to ignite carbon with consequent supernova explosion or because of mass loss by stellar wind the whole envelope is removed and the star becomes a white dwarf. Therefore, from the standpoint of the evolution of AGB stars, mass loss plays an important role. This may occur in two forms: a stationary wind, whose rate has been approximated by various relationships involving basic stellar parameters like luminosity, mass, radius, etc, the most popular of which is the Reimers (1975) rate, and a so-called superwind which leads to the ejection of a planetary nebula shell (see Renzini \& Voli 1981; Iben \& Renzini 1984). From the standpoint of 
classical Cepheids, the AGB phase has no relevance, the only exception being the possibility that under favourable circumstances, past core He-burning and prior to the start of the thermally pulsing regime, during the so-called early AGB, a second blue loop develops. This is due to the complicated interplay between the contracting $\mathrm{C}-\mathrm{O}$ core, the He-burning shell, and the structure of the outer envelope. The time scale of this crossing is about $10^{3} \mathrm{yr}$.

\subsection{Nuclear reactions}

Although the rates of the major reactions involved in $\mathrm{H}$ - and He-burning are sufficiently known, the work of Fowler et al. (1967, 1975) and Harris et al. (1983) shows that many of them have changed over the years and that some uncertainty is possible. This is particularly true for the ${ }^{12} \mathrm{C}(a, y){ }^{16} \mathrm{O}$ reaction whose cross section has been recent ly remeasured and increased by approximately a factor of five (Kettner et al. 1982) with respect to the Fowler et al. (1975) estimate. Although this rate has been somewhat reduced by the study of Fowler (1984), only a factor of three higher, settlement of this question has not yet been achieved. The presently accepted increase with respect to the classical value is a factor of 2 to 3 . Nonetheless, it has been known for a long time (Iben, 1972) that any uncertainty in the rate of this reaction will immediately reflect on the evolutionary behavior of stellar models that become Cepheids. In fact, the larger the cross section, the greater is the extent to which carbon is converted into oxygen, and the longer the core He-burning lifetime. The greater the cross section, the further the H-exhausted core increases under the action of the H-burning shell, and the further the loop extends to the blue before rapid core contraction and envelope expansion set in and the evolution proceeds back to the red. As a result, for a given chemical composition, the blue band of core He-burning models turns out to be more inclined toward the blue than with the old value, since the shift to the blue tends in fact to be greater at higher masses. How this would affect the intersection with the instability strip is obvious. However, the effect of increasing the rate of ${ }^{12} \mathrm{C}(\boldsymbol{a}, \mathbf{y}){ }^{16} \mathrm{O}$ is marginal toward the low end of intermediate mass stars and in the range of massive stars, where in these latter it is wiped out by the overwhelming effects of mass loss.

\subsection{Radiative opacity}

Among the various aspects of the input physics of model calculations, radiative (and conductive) opacities still suffer from a great deal of uncertainty, and to even small changes of which the properties of stellar models are extremely sensitive. To clarify the role played by each particular source of opacity to the building up of the total radiative opacity, Iben \& Renzini (1984) have distinguished several temperature ranges (which are also somewhat dependent on the actual densities encountered in the stellar interiors), which are briefly summarized here. (i) At very low temperatures $\left(<2.5 \times 10^{3} \mathrm{~K}\right)$, the opacity is dominated by transitions in molecular levels in species such as $\mathrm{CO}, \mathrm{TiO}, \mathrm{H}_{2} \mathrm{O}, \mathrm{CN}, \mathrm{CH}$ etc. (ii) In the range $2.5 \times 10^{3}$ to $5 \times 10^{3} \mathrm{~K}$, the major contribution to opacity comes from $\mathrm{H}^{-}$, the number of required 
electrons being controlled by the abundances of elements of low ionization potential such as $\mathrm{Mg}$, $\mathrm{Si}$; (iii) In the range $5 \times 10^{3}$ to $5 \times 10^{5}$ the opacity is mostly given by bound-bound, bound-free and free-free transitions of $\mathrm{H}$ and He. (iv) At temperatures in the range $5 \times 10^{5}$ to $5 \times 10^{6} \mathrm{~K}$, the bound-bound and bound-free transitions of elements from carbon to iron (shortly indicated as the CNO group of elements) dominate the opacity. (v) Finally, at temperatures higher than $5 \times 10^{6} \mathrm{~K}$, the opacity is simply given by electron scattering. While the opacities of regions (iii) and (v) are probably not significantly in errors, those of regions (i) and (ii) are far more uncertain. However, these latter regions are always associated with convection, and their uncertainty is simply overwhelmed by the uncertainty in the treatment of convection. Region (iv) is by far the one presenting the major difficulty. In this case, the high number of electrons in each elemental species, the large number of ionization and excitation stages, the non-hydrogenic structure of the electronic configuration, the distortions of this induced by nearby electrons and ions that are difficult to model, all conspire to make the opacity of this temperature range very difficult to calculate, and of course, with the highest degree of uncertainty. This is the main reason why the characteristics of stellar models that are very sensitive to the so-called middle temperature opacity are still highly uncertain and a matter of debate. We will touch upon this topic in the course of this review.

\subsection{Mass loss by stellar winds}

To our knowledge, the effect of mass loss by stellar wind along the RGB phase on stars of intermediate mass and on Cepheid stars, in particular, has never been systematically investigated. The early studies of Lauterborn et al. (1971), and Lauterborn \& Siquig (1974) have shown that mass loss may be able to suppress the formation of blue loops if more than $10 \%$ for a $5 \mathrm{M}_{\odot}, 13 \%$ for a $7 \mathrm{M}_{\odot}$ and $20 \%$ for a $9 \mathrm{M}_{\odot}$ star of the initial mass is lost. Although the results are expected to depend somewhat on the initial chemical composition and details of the input physics, the implication is that a moderate degree of mass loss in intermediate mass stars can reduce the number of crossings from a few (say three) to only one (the first). With the current rates of mass loss (see Reimers 1975; Lamers 1981; de Jager et al. 1988) for red giant stars in the luminosity range of interest here, and the lifetime of the RGB typical of an intermediate mass star, mass loss is likely not to be of much importance. This is particularly true for classical models. The above conclusion no longer holds for Cepheids whose initial mass is greater than say $15 \mathrm{M}_{\odot}$, where mass loss may be important and it is already included in most model calculations (see Chiosi \& Maeder 1986). Nonetheless, on one hand the observational determinations of mass loss rates are still very uncertain and tending to grow, and their dependence on basic stellar parameters (luminosity, mass, radius, etc.) poorly known, on the other hand stellar models potentially undergoing mass loss (along the RGB) have changed with respect to the classical ones for which the estimate of the effect of mass removal has been made. This means that the real amount of mass that can be lost by intermediate mass stars during their RGB phase is still highly uncertain. The 
calculations of Bertelli et al. (1985) for Pop I intermediate mass stars $(z=0.02)$ with initial mass $5 \mathrm{M}_{\odot}$ and $9 \mathrm{M}_{\odot}$, in which different algorithms for the mass loss rates have been adopted, clarify the role played both by the algorithms themselves and the underlying evolutionary models. The following relations for the mass loss rate have been tested

$$
\begin{aligned}
& \dot{M}=\mathbf{a} 1.27 \times 10^{-5} \mathrm{M}^{-1} \mathrm{~L}^{1.5} \mathrm{~T}_{\text {eff }}^{-2} \\
& \dot{\mathrm{M}}=\mathbf{n} 0.11 \mathrm{~L}^{1.96} \mathrm{~T}_{\mathrm{eff}}^{-3.54}
\end{aligned}
$$

where $\mathrm{M}, \mathrm{L}$ are in solar units, $\dot{\mathrm{M}}$ in $\mathrm{M}_{\odot} \mathrm{yr}^{-1}$, and relation (8) is the Reimers (1975) law, whereas relation (9) is adapted from Waldron (1984). The parameter $\boldsymbol{\eta}$ has a well known meaning, whereas the parameter a has been introduced by Bertelli et al. (1985) to reconcile the rates from the two laws in the domain of massive stars $(a=1$ yields rates equal to those for $\eta=1$ ). In classical models, little mass is lost during the RGB phase independently from the adopted algorithm, thus confirming the older studies quoted above (see the data in Tables $2 \mathrm{a}$ and $2 \mathrm{~b}$ of Bertelli et al. 1985). On the contrary, in models with convective overshoot (to be discussed below), owing to their much higher luminosity, the amount of mass lost during the whole core He-burning phase critically depends on the adopted algorithm and the initial mass of the star. Stars of about $5 \mathrm{M}_{\odot}$ and lower, independently of the algorithm for the mass loss rate, lose only a few per cent and evolve in the HR diagram in a way similar to that of classical models. Stars of higher mass depending on the adopted algorithm, may lose an enormous amount of mass and accordingly drastically change their path in the HR diagram (see the data of Table $2 a$ and $2 b$, and Figures $11 a$ through $11 c$ of Bertelli et al. 1985). This simply means that the problem of mass loss from intermediate mass stars is far from being settled and more work is needed. Another interesting possibility has been advanced by Willson (1987) and Willson \& Bowen (1984), who suggest that in order to reduce the evolutionary masses to better agree with the pulsational masses (see below), and to avoid the difficulties encountered when mass loss occurs during the RGB phase, mass loss, somehow triggered by the pulsational instability itself, should occur while the star is within the instability strip. Evolutionary calculations including mass loss during the Cepheid stage confirm that Cepheids are trapped in the instability strip, mass loss can continue over a relatively long time scale, the total mass is significantly reduced, whereas the luminosity is about constant (Brunish \& Willson, 1987). The rates of mass loss required by these model calculations are of the order of $7 \times 10^{-9} \mathrm{M}_{\odot} \mathrm{yr}^{-1}$ for a $5 \mathrm{M}_{\odot}$ star and $2 \times 10^{-7} \mathrm{M}_{\odot} \mathrm{yr}^{-1}$ for a $7 \mathrm{M}_{\odot}$ star. Unfortunately, efforts to observe Cepheid winds directly have so far given inconclusive results. Finally, it is worth recalling that mass loss not only affects the mass and the path in the HR diagram of a star, but also its appearance as a Cepheid. In fact, on one hand the mass is decreased (even though by a few per cent according to the classical algorithm of Reimers (1975), on the other hand the period-luminosity relation is changed. At given luminosity, the period is expected to increase at decreasing mass as a consequence of the period-mean density relationship (see the study by Stift (1984) on the frequency-period distribution). 


\subsection{Rotation and duplicity}

Rotation. Main sequence progenitor stars of Pop I Cepheids rotate at speeds of about $250 \mathrm{~km} \cdot \mathrm{sec}^{-1}$ (Slettebak 1970), whereas red giant stars have low rotational velocities (Kraft 1970), implying that angular momentum is either lost or more likely transferred to inner layers of the star. Owing to the complexity of including rotation in stellar model calculations, since first 2D or 3D codes and second modelling of angular momentum transfer and coupling of it with convection are required, rotation is usually neglected in most of the stellar models currently available in literature. Despite the complexity of the problem, a few pioneering calculations exist (Kippenhahn et al. 1970; Meyer-Hofmeister 1972; Endal \& Sofia 1976, 1978 , 1979) showing from the standpoint of one dimensional calculations the effect of rotation on the evolution of intermediate mass stars. Somewhat contradictory results are obtained. While, they all agree that the core He-burning lifetime is lengthened by rotation, they disagree in the properties of the blue loop. Endal \& Sofia $(1976,1978)$ find that the luminosity and tip effective temperature of the blue loop is increased and decreased, respectively, whereas the opposite is found by the other authors. The reasons for the disagreement cannot easily be singled out.

Duplicity. A large fraction (up to about 308) of Pop I Cepheids are members of binary systems, with companions of similar mass. Depending on the separation, these stars can either evolve as if they were single objects (larger than a few $100 \mathrm{R}_{\odot}$ ) or mutually influence their structure and evolution by mass exchange. Unfortunately, very few calculations of binary systems of intermediate or massive stars are available in the literature, so little can be said on this topic. Likely, if a star fills its Roche lobe as a red giant, it will probably lose enough mass to prevent it from evolving further into the instability strip. Therefore this star can appear as a Cepheid only during its first crossing. The accreting mass companion will certainly evolve in a manner different from that of a single star having the companion's star initial or current mass. If this star crosses the instability strip, how the different evolutionary history would affect its appearance as a Cepheid cannot be foreseen (see Becker 1985).

\section{EVOLUTION WITH CONVECTIVE OVERSHOOT}

It has already been recalled that starting from the core H-burning stages the extension of convective cores in massive stars are affected by the H-semiconvection caused by the high radiation pressure and electron scattering opacity, whereas those of intermediate and low mass stars are affected by the He-semiconvection driven by an opacity difference between carbon-rich and carbon poor mixtures during the core He-burning phase. However, in addition to the problem of semiconvection, there is a more fundamental question concerning the mass extension of convective cores, i.e. the amount of convective overshoot from their borders. This in fact is always expected to occur at the boundary of a formally convective core, where the kinetic energy of convective elements carries them a finite distance into layers which are 
formally stable against convection. In other words, the classical criterion used to set the boundary of a core (the layer where the radiative and adiabatic temperature gradients are equal) simply means that the acceleration imparted to convective elements by the buoyancy forces becomes zero, whereas their kinetic energy does not. It goes without saying that the correct condition would be the one requiring the vanishing of the kinetic energy. In such a case further mixing may arise between the convective core and a part of its neighbouring radiative region causing a net increase in the mass extension of the fully mixed region. Though simply motivated from the standpoint of physical arguments, great controversy exists on the exact amount of convective overshoot. According to the theoretical formulations of this phenomenon proposed by different authors, the overshoot distance at the edge of the formally convective core goes from zero to about 2 pressure scale heights (see Chiosi 1986 for a review of the subject and exhaustive references). In any case, convective overshoot gives rise in stellar models to differences in structure and evolution that are much more substantial than those given by semiconvection. In addition to the above arguments of a merely theoretical nature, there are many observational facts requiring a deep revision of the basic physics, such as convective cores significantly bigger than usually assumed. Among others we recall:

a) The width of the main sequence band, which appears to be larger than predicted by standard models, and the number ratio of evolved to unevolved stars in galactic clusters (Maeder \& Mermilliod 1981) and in the populous young globular clusters, like NGC 1866 in LMC (Becker \& Mathews 1983; Chiosi et al. 1988), which is larger than expected. b) The anomalous behaviour of several old open clusters, which in spite of a turnoff mass of about $1.5 \mathrm{M}_{\odot}$ do not exhibit an extended RGB indicating that the core He-flash does not occur but, on the contrary, He-burning takes place quietly (Barbaro \& Pigattto 1984).

c) The lack of very luminous AGB stars in several clusters of the Magellanic Clouds, where they are expected on the basis of the turnoff mass. This fact suggests that $M_{u p}$ is much lower than predicted by classical models.

d) The long lasting discrepancy between evolutionary and pulsational mass together with the frequency-period distribution of Cepheid stars (see below).

\subsection{Stellar models with convective overshoot}

Among the possible formulations of convective overshoot, we have followed the method developed by Bressan et al. (1981), which is based on a non-local view of convection, uses the mixing length theory, and takes the mean free path of convective elements as a parameter to be fixed by comparing model results with the observational properties of star clusters. The mean free path is given by $l=\lambda_{H_{p}}$, where $H_{p}$ is the local pressure scale height and $\lambda$ is a free parameter. The evolutionary models we are going to describe are for $\lambda=1$, which has been found to give the most interesting results (Bertelli et al. 1985; 1986a, b; Bressan et al. 1986). In these models the rate of the ${ }^{12} \mathrm{C}(\mathrm{a}, \mathrm{Y}){ }^{16} \mathrm{O}$ reaction is assumed to be three times greater than the classical value 
of Fowler et al. (1975) as indicated by the recent measurements of the cross section (see Kettner et al. 1982).

i) The core H-burning phase. Stars whose initial mass is high enough to develop a convective core on the main sequence, $\left(M>1.1 \mathrm{M}_{\odot}\right)$, are strongly affected by convective overshoot. In fact, owing to their more massive convective cores, they run at higher luminosities and live longer than classical models. They also extend the main sequence band over a wider range of effective temperatures, this trend increasing with the stellar mass. Massive stars $\left(M>40 \mathrm{M}_{\odot}\right)$ would spread all across the colour-magnitude (CM) diagram, were it not for the contrasting effect of mass loss (see Bressan et al. 1981).

ii) The core He-burning phase in massive and intermediate mass stars. The overluminosity caused by overshoot during the core H-burning phase still remains during the shell $\mathrm{H}$ - and core He-burning phases. The mass of the H-exhausted core, $\mathrm{M}_{\mathrm{He}}$, and the mass of the $\mathrm{C}-\mathrm{O}$ rich, He-burning convective core, are increased by overshoot. However, as a consequence of the higher luminosity, the lifetime of the He-burning phase $\left(t_{\mathrm{He}}\right)$ gets shorter in spite of the increase in the core mass. This, combined with the longer $H$-burning lifetime, $t_{H}$, makes the ratio $t_{H e} / t_{H}$ fairly low (from 0.12 to 0.06 when the stellar mass varies from 1.6 to $9 \mathrm{M}_{\odot}$ ). The location of the core He-burning models in the $\mathrm{CM}$ diagram can be schematically summarized as follows. In massive stars, where mass loss dominates throughout the stellar life, the He-burning phase takes place entirely in the blue for $M>60 \mathrm{M}_{\theta}$, partly in the blue and partly in the red for stars in the range 60 to $20 \mathrm{M}_{\theta}$ or thereabouts, the lifetime of the two sub-phases being controlled by the mass loss rate. The blue loops of intermediate mass stars $\left(\mathrm{M}<\mathrm{M}_{\mathrm{HeF}}\right)$ are much less extended, whereas their dependence on chemical composition and nuclear reaction rates is the same as in classical models. This implies that the blue band of core He-burning models is less inclined in the HR diagram and, therefore, for a given chemical composition the intersection with the instability strip occurs at different luminosities, hence masses. Figure 2 shows the predicted locations in the HR diagram of stars of Pop I chemical composition $(Y=0.28$ and $Z=0.02)$ calculated with convective overshoot $(\lambda=1)$.

iii) The core He-burning phase of low mass stars. HB stars while burning helium in the centre, possess a convective core whose mass extension may be affected by convective overshoot. Models of HB stars have been calculated according to the Bressan et al. (1981) algorithm with $\lambda=1$, core mass equal to $0.475 \mathrm{M}_{\odot}$ and total mass in the range 0.6 to $1.4 \mathrm{M}_{\odot}$ (Bressan et al. 1986). The most important result of these calculations is that in presence of convective overshoot, semiconvection and/or breathing pulses of convection (see Castellani et al. 1985 for details) never develop. In particular, these models predict lifetime ratios in $\mathrm{RGB}, \mathrm{HB}$ and $\mathrm{AGB}$ that are in closer agreement with the observational ones derived from star counts in globular clusters (see Buonanno et al. 1985, for a discussion of this topic). A detailed description of these models and a comparison with the observational data for globular clusters are given in Bressan et al. (1986) to whom we 
Figure 2. The HR diagram of models with convective overshoot $(\boldsymbol{\lambda}=1)$, standard radiative opacities, and chemical composition $Y=0.28$ and $Z=0.02$. The hatched areas show the phases of slow nuclear burning. No mass loss along the RGB phase of intermediate mass stars is taken into account. The value of the initial mass is indicated on the zero age main sequence. Note the much wider main sequence band and the less inclined blue band of He-burning models produced by the effect of convective overshoot. The instability strip is also drawn, and three characteristic luminosities at the intersection with the blue band of core He-burning models are shown (see text).

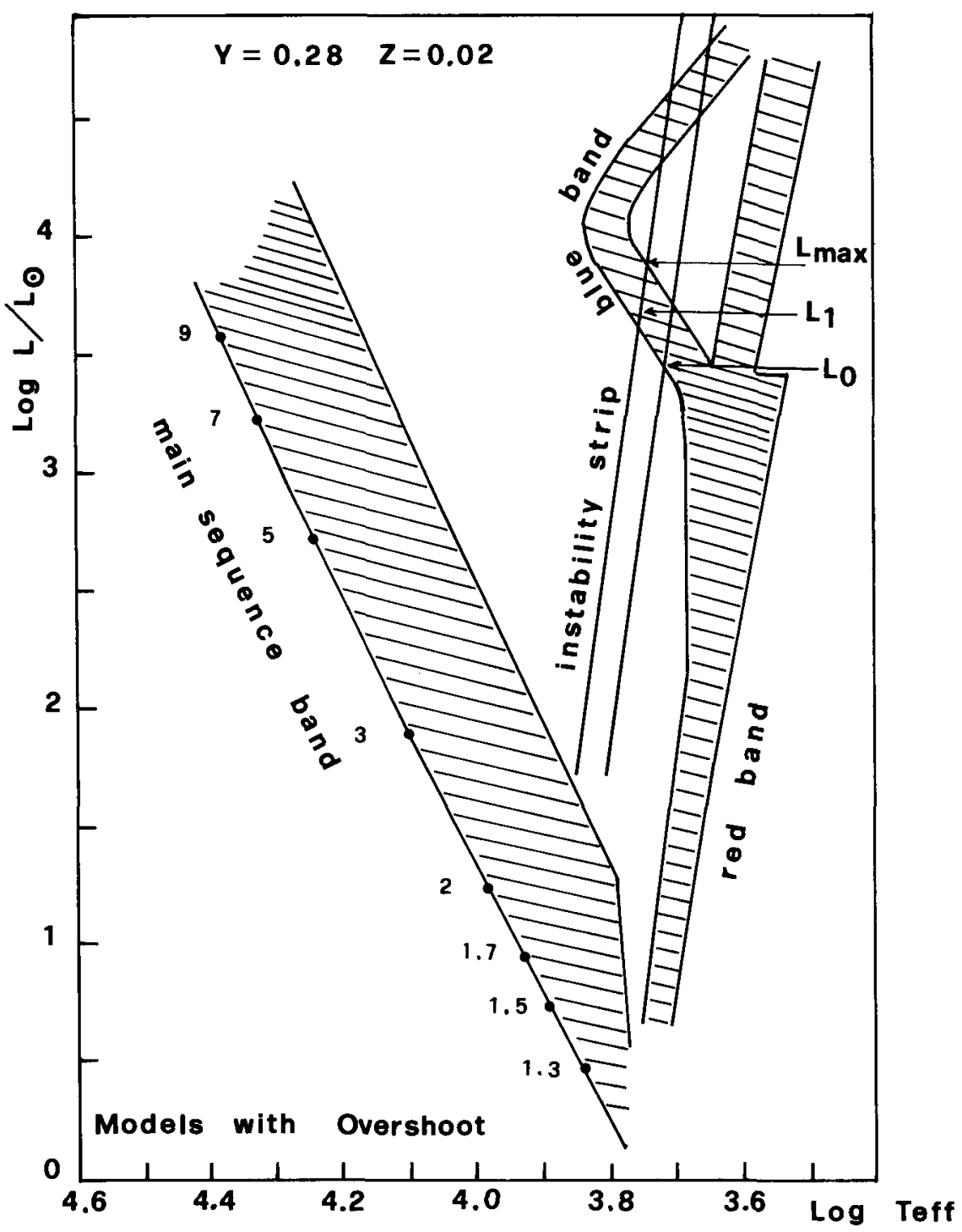


refer.

iv) The critical masses $\underline{M}_{\text {mas } \perp} M_{u p}$ and $\underline{M}_{H e F}$. By virtue of the larger masses of the He and C-O core left over at the end of core $H$ and He-burning phases respectively, the relationships between the initial mass and the mass of the He and $\mathrm{C}-\mathrm{O}$ core, which define the critical masses in question are different in models with convective overshoot. The most important result is that now both $\mathrm{M}_{\mathrm{up}}$ and $\mathrm{M}_{\mathrm{HeF}}$ are significantly lower than in classical models. The new masses are given in Table 1 for two initial chemical compositions. This means that no $A G B$ and no prolonged RGB phases are expected for stars of initial mass above the new $\mathrm{M}_{\text {up }}$ and $\mathrm{M}_{\text {HeF }}$, respectively. The impact of this result on the observational front is straightforward and of paramount importance.

v) The AGB phase. Over the past few years, the evolution of AGB stars has been the subject of many theoretical.studies, recently reviewed by Lattanzio (1988a), aimed at understanding why carbon stars can be formed at lower Iuminosities than predicted by the classical models of Iben (1975a,b, 1976). The problem came when a comparison between theoretical AGB star distributions (Iben 1981; Renzini \& Voli 1981) and observations of Magellanic Cloud stars (Blanco et al. 1978, 1980) showed that no carbon stars were expected less luminous than $M_{B}=-5$ or brighter than $M_{B}=-7.5$, in contrast with the observations indicating that carbon stars occur in the range $-3.5<\mathrm{M}_{B}<-6$. Since the evolution along the thermally pulsing AGB phase is essentially governed by the linear relationship between the H-exhausted core mass, or equivalently the $\mathrm{C}-\mathrm{O}$ core mass, and the luminosity (see Iben \& Renzini 1983 for all details), this is equivalent to say that the third dredge up must operate at lower core masses than suggested by the current theory. The series of recent models by Lattanzio (1986, 1987a, 1987b, 1988), Boothroyd \& Sackmann (1988a-d) and Hollowell (1987, 1988) in the mass range 1-3 $\mathrm{M}_{\odot}$, in which revised algorithms for semiconvection and breathing convection, the use of better opacities, the inclusion of mass loss by stellar wind, and the estimate of the dependence of the $\mathrm{C}-\mathrm{O}$ core mass at the first thermal pulse on the initial chemical composition and initial mass of the star, concur to succeed in producing models of carbon stars of quite low Iuminosity. Models incorporating convective overshoot (see Chiosi et al. 1987) have not yet been evolved into the thermally pulsing regime, however we may foresee that they should give similar results, since they share many features in common with models calculated with semiconvection and breathing convection. Among other things, since they predict that $M_{u p}$ is much lower than the classical value, this would help removing all

Table 1

\begin{tabular}{lllll}
\hline $\mathrm{X}$ & $\mathrm{Z}$ & $\overline{\mathrm{M}}_{\text {mas }}$ & $\mathrm{M}_{\text {up }}$ & $\overline{\mathrm{M}}_{\text {HeF }}$ \\
\hline 0.700 & 0.020 & 9 & 6 & 1.6 \\
0.700 & 0.001 & 8 & 5 & 1.6 \\
\hline
\end{tabular}


very bright $A G B$ stars as indicated by the observational data (very few $A G B$ stars brighter than $M_{B}=-6$ ).

\subsection{Clusters of the LMC: NGC 1866 a test for convective} overshoot

Although stellar models calculated with semiconvection and breathing convection look similar to those calculated with convective overshoot, the two types of model differ profoundly in many quantitative details. The characterizing features are the core $\mathrm{H}-$ and He-burning lifetimes and evolutionary brightening from the main sequence to the most advanced stages. These should immediately reflect on the morphology of CM diagrams of star clusters, namely the number of evolved to main sequence stars and associated luminosity functions. It goes without saying that the ideal laboratory where theories of stellar structure, in particular those concerning the domain of massive and intermediate mass stars, can be tested are the rich young clusters of the Magellanic Clouds. These in fact by virtue of their large number of stars allow statistically meaningful comparisons between theory and observations in samples of about coeval and chemically homogeneous objects even for the shortest lived evolutionary stages. To this purpose, Chiosi et al. (1988) have taken accurate Johnson BV CCD photometry of the cluster NGC 1866 of the Large Magellanic Cloud (LMC) and compared its $\mathrm{CM}$ diagram and main sequence star Iuminosity function with the theoretical predictions based on both classical models and models with convective overshoot. The results of the chiosi et al. (1988) study are briefly summarized below.

NGC 1866 is a rich young cluster situated at about $2^{\circ}$ North of the LMC centre, whose total mass is estimated in the range 3.6 to $8.5 \times 10^{4} \mathrm{M}_{\odot}$ (Heckman 1976). It is classified as a type III in the Searle et al. (1980) system, with integrated apparent visual magnitude $V=9.73$, and colours $(B-V)=0.25$ and $(U-B)=-0.04$ (van den Bergh 1981) and colour excess $E(B-V)=0.07$ (Persson et al. 1983). Johnson $B$ and $V C C D$ photometry of 1517 stars in the central region of NGC 1866, and of 640 stars in a nearby field have been obtained with the RCA \#5 CCD on the ESO $2.2 \mathrm{~m}$ telescope and reduced using the ESO version of DAOPHOT. TO define the region of the cluster frame that suffers from the least degree of uncertainty due to photometric incompleteness and contamination by field stars the following procedure was adopted. First, a composite CCD frame (cluster and field) was obtained superposing a few bright stars in common, and an iterative procedure was used to fix the cluster centre. Second, the surface brightness profile of the cluster was generated in a way similar to Meylan \& Djorgovski (1987) and compared to the theoretical density profiles calculated from multi-mass, King-Michie models (Meylan 1987a,b, 1988) in the case of the Salpeter law with slope $\mathrm{x}=2.35$ and isotropical velocity dispersion. The cluster turned out to possess a concentration $c=\log r_{t} / r_{c}=1.5$, a core radius $r_{c}=6 ! 69$ and a tidal radius $r_{t}=211: 6$. Furthermore, since the last point of the observed profile is at about $r=2: 40$ to which a density contrast of about a factor of $10^{3}$ corresponds, the radius $r_{W}=2 ? 45$ is taken as the limiting radius beyond which the majority of 
stars are field objects. Third, the surface brightness profile was reconstructed from the photometric data ( $V$ and/or $B$ magnitudes) of individual stars falling within circles of increasing radius centred on the cluster centre. The simultaneous comparison of the surface brightness profile, the density profile, and the surface brightness profile reconstructed from individual stars, shows that the ring confined between 0.52 and 0.98 is the one suffering from the least degree of incompleteness by crowding and other causes, and it can be taken to represent the cluster population.

The $\mathrm{CM}$ of the stars falling in the ring is assumed as the reference $\mathrm{CM}$ to which theoretical models ought to be compared. This is shown in Figure 3. In this CM diagram, three regions of particular interest can be defined, namely: the region of the main sequence stars (MS), the region of red giant stars brighter than 17 th magnitude in $V$ (BRGS), the region of red giant stars fainter than 17 th magnitude in $V$ (FRGS). The total number of stars is 656, of which MS $=594$, BRGS $=39$ and FRGS $=$ 23. This $\mathrm{CM}$ diagram is photometrically complete to $\mathrm{V}=19.2$. The field is given by the region of the composite CCD frame outside $r_{W}$ whose area happens to be nearly identical to that of the cluster ring. After correction for photometric completeness (crowding) and contamination by field stars by means of the zapping technique of Mateo \& Hodge (1986), the total number of stars is 1020 made of MS $=954$, BRGS $=41$, FRGS $=$ 25. The group of BRGS likely represents the population of evolved stars belonging to the cluster, i.e. genetically linked to the populations of

Figure 3. The CM Diagram of the selected ring of NGC 1866. Capital letters $A, B$ and $C$ indicate three groups of stars discussed in the text.

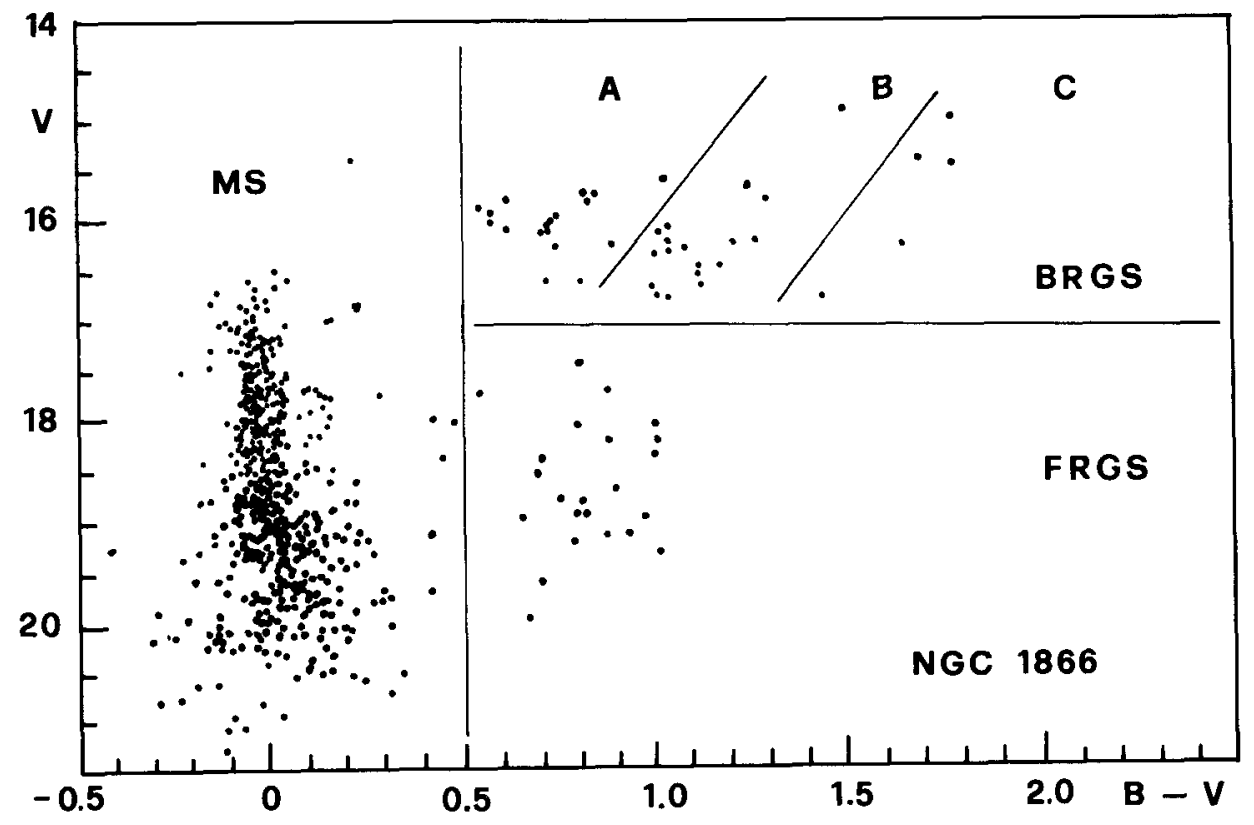


MS, whereas FGRS are likely field stars. NGC 1866 contains also several Cepheids: 11 well established objects (Walker 1987, and references therein) and about 10 more recent candidates (Storm et al. 1988), which are located in the middle of BRGS. The sample of BRGS can be split into three subgroups (labeled A, B and C). Group A and B contain almost equal numbers of stars and are located at the blue and red side of the instability strip, whereas group $C$ is populated by stars (very few indeed) of the same luminosity but of much redder colour. Likely, these do not belong to the same population as those of groups $A$ and $B$, but they are probably genetically related to FRGS.

The luminosity function for the MS stars is presented in Figure 4. This is the integrated number of stars counted from the tip of the main sequence band down to the current magnitude bin and normalized to the number of BRGS. The advantage of this type of luminosity function is that it can be simply related to the ratio of core $\mathrm{H}$ - to core He-burning lifetime.

On the theoretical side, a computer code has been developed for constructing synthetic CM diagrams, luminosity functions, integrated magnitudes and colours in the UBVRI bands as a function of the age, chemical composition, initial mass function, total number of stars, given number of evolved stars corresponding to the observed BRGS, and finally stellar evolution input. This algorithm also allows for

Figure 4. The integrated luminosity function for the main sequence stars normalized to the number of bright red giant stars ( $<<17)$ likely representing the evolved counterpart of the main sequence population (see text for more details).

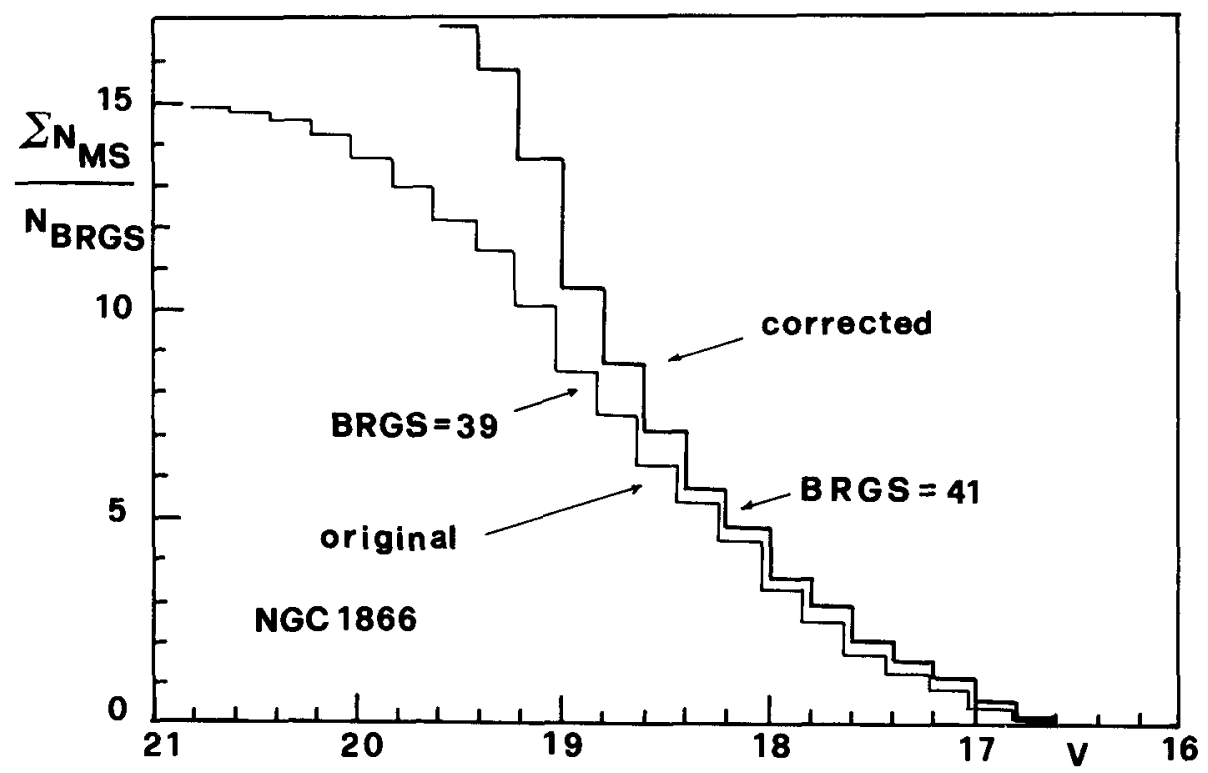


dispersion in age and fluctuations of a stochastic nature in the initial mass function.

The comparison between theory and observations based on several fiducial characteristics of the the CM diagram, such as the mean location of the main sequence band, its terminal luminosity, the mean luminosity of the blue and red giant stars, the tip of the blue loop (if present), and the location of the reddest stars (Hayashi limit) allows us to derive the age and chemical composition and to estimate the distance modulus in the two alternatives for the stellar evolution, namely classical models and models with overshoot. The simultaneous fit of the above constraints indicates that the appropriate chemical composition is $Y=0.28$ and $Z=$ 0.02 (or slightly less say $z=0.010-0.015$ ), the true distance modulus is $(\mathrm{m}-\mathrm{M})_{0}=18.6$ in good agreement with the value determined from the near infrared photometry of Cepheid stars (Welch et al. 1987), and the age is $200 \times 10^{6}$ yr for models with overshoot, or $70 \times 10^{6}$ yr for classical models. However, with these latter models the fit of the red giant stars is rather poor, as they turn out to be fainter than the mean luminosity of the 1oop and bluer than the Hayashi limit. Other combinations of age and chemical composition can be neglected as they would lead either to a very poor fit of the global properties of the CM diagram or to unacceptably low distance moduli. While the CM diagram does not clearly indicate the underlying evolutionary scheme, can be seen by looking at the integrated main sequence luminosity function normalized to the number of post main sequence stars. The comparison with the observational luminosity function strongly favours models with convective overshoot. In other words, the star counts in the clusters suggest that the ratio of core $\mathrm{H}$ - to He-burning lifetimes and the range of luminosity spanned by main sequence stars, whose masses are compatible with those of the evolved stars, must be smaller and wider, respectively, than given by the classical models. This is possible only if more efficient mixing occurs all over a star's evolutionary history and not only in stages beyond the main sequence phase. It is worth emphasizing that other mixing processes like semiconvection and/or breathing convection, would not be able to satisfy the observational demand, since they tend to increase the number of evolved stars with respect to those of the main sequence. Agreement with the classical models is marginally possible only by increasing the slope of the initial mass function above 4.

\section{THE MASS-DISCREPANCY OF CEPHEID STARS}

Since the review on cepheid masses by Cox (1980) is still fully relevant, we will not go into any detail here. It has long been debated whether the masses determined from stellar evolution theory (L-M relation) agree with those derived from pulsation theory ( $\mathrm{P}-\mathrm{M}-\mathrm{L}-\mathrm{T}_{\mathrm{eff}}$ relation) (see Iben, 1974; Iben \& Tuggle, 1972, 1975). In general, pulsational masses ( $\mathrm{M}_{\mathrm{pul}}$ ) are estimated to be 30 to $40 \%$ lower than evolutionary masses ( $\mathrm{M}_{\text {evol }}$ ) of the same luminosity. The apparent disagreement between $M_{\text {evol }}$ and $M_{\text {pul }}$ has been interpreted as an indication of 1) significant mass loss at some point between the main sequence and the Cepheid stage, 2) systematic errors in the 
determination of the distance scale with respect to the classical values of Sandage \& Tammann (1969), possibly an underestimate of the distance to the Hyades, and in the derivation of the $\mathrm{T}_{\text {eff }}$ assigned to a Cepheid, 3) errors in the pulsation theory and 4) uncertainties in model calculations (particularly as these are influenced by uncertainties in opacity).

Cox (1980), in his review, has distinguished six methods of determining the mass of Cepheids: 1) the evolutionary mass (Mevol), 2) the theoretical mass ( $\left.M_{\text {the }}\right)$, introduced by $C o x$ et $a l$. (1977) and based on the simultaneous solution of four equations for the unknowns $M, R$, $I$ and $Q_{i}$, where $Q_{i}=P_{i}(M / R)^{1 / 2}$, 3) the pulsational mass $\left.\left(M_{\text {pul }}\right), 4\right)$ the bump Cepheid mass $\left(M_{\text {bump }}\right)$, 5) the double and triple mode mass ( $\left.M_{\text {beat }}\right)$ and 6 ) the Baade-Wesselink radius mass $\left(\mathrm{M}_{\mathrm{BW}}\right)$. The bump masses are calculated by modeling the Hertzprung progression (dependence on period of a secondary maximum in the light curves of Cepheids with period $\mathrm{P}_{0}$ in the range 6 to 18 days). They require good light curves and phase coverage. The beat masses are derived from the periods $P_{0}$ and $P_{1}$ (fundamental and first overtone) by means of mass luminosity relations as functions of $\mathrm{P}_{1} / \mathrm{P}_{0}$, which are very sensitive to the physical properties of models producing them. Finally the Baade-Wesselink radius masses are obtained from the $Q_{i}\left(P_{i}, M, R\right)$ relations, where $Q_{i}$ are from theory, $P_{i}$ from observations, $R$ is the radius measured by the Baade-Wesselink method. It goes without saying that each method is affected by many uncertainties inherent both to the observational and theoretical quantities required to derive the mass.

Cox (1980) discusssing the available data came to the conclusion that the "primary" mass problem ( $\mathrm{M}_{\text {evol }}>\mathrm{M}_{\text {pul }}$ ) could be solved by varying the intrinsic luminosity of Cepheids (longer distance modulus) and by lowering the Cepheid temperatures with improved reddening and temperature calibrations. According to $\mathrm{Cox}, \mathrm{M}_{\text {evol }}$ is normally in agreement with all the others but the bump Cepheid masses and the double and triple mode Cepheid masses. However, recent developments on the subject somewhat weaken Cox's conclusion. First, even though the Haydes distance modulus has been increased to $3.4 \mathrm{mag}$ (Hanson, 1979;

Vandenbergh \& Bridges 1984), there is the well known complication related to the Hyades zeropoint in the distance scale, in that it remains uncertain how the distance to other clusters should be corrected for the effect of the high metallicity of the Hyades. This would immediately affect the distance hence absolute visual magnitude assigned to individual Cepheids. Second, the thorough discussion by Pel (1985) on the fundamental parameters of Cepheids points out that they are still far from being fully assessed, and that the problem of the mass discrepancy, albeit not very large, is nevertheless significant (see the cases of $U$ Sgr and $S$ Nor for which the use of revised data has again moved $M_{\text {evol }}$ and $M_{\text {pul }}$ apart). Finally, Simon (1986) and Böhm-Vitense (1986) have rediscussed the problem of the beat and bump Cepheid masses. Using the Schmidt (1984) distance scale, along with Baade-Wesselink masses, Böhm-Vitense (1986) finds the following relationships among the various masses: for $\mathrm{P}>6$ days, $M_{\text {evol }}>M_{B W}=M_{\text {pul }}>M_{\text {bump }}$, whereas for $\mathrm{P}<6$ days, $\mathrm{M}_{\text {evol }}>\mathrm{M}_{\mathrm{BW}}>\mathrm{M}_{\text {pul }}>\mathrm{M}_{\text {beat }}$. Simon $(1982,1987)$, examining 
the period ratios $\left(P_{1} / P_{0}\right.$ for beat Cepheids and $P_{2} / P_{0}$ for burp Cepheids), points out that not only are the masses discrepant but also the observed period ratios are lower than those predicted by the theory. While the discrepancy in the period ratios can be corrected by lowering the mass of a model at given luminosity, the period ratios are also very sensitive to the interior structure. The $\mathrm{M}_{\text {beat }}$ and $\mathrm{M}_{\text {bump }}$ could be raised to $M_{\text {evol }}$ only by accepting that the current model structure is varied for instance by changes in the opacity law such as an increase of a factor of 2 to 3 in the middle temperature region (see below). It transpires from the above discussion that $\mathrm{M}_{\text {evol }}$ is usually taken as the standard of comparison for the various mass determinations despite the fact that it is perhaps the most difficult to obtain, since it requires the observational determination of $\mathrm{P}, \mathrm{L}$ or $\mathrm{T}_{\text {eff }}$ and the assumption of $a$ mass-luminosity relationship from stellar models. Among the various causes for the mass discrepancy, substantial mass loss along the RGB phase and/or uncertainties or differences in the evolutionary schemes have been advocated. We have already pointed out that under the current mass loss rates and their parameterizations, little mass can be lost, certainly insufficient to remove the discrepancy in question. The adoption of unusually high rates of mass loss, on one hand may solve the problem, but on the other hand can give rise to devastating effects on the evolutionary behaviour of intermediate mass stars thus making hard to interpret even the overall properties of Cepheid stars. If mass loss is supposed to be negligible, one way out of the problem of mass discrepancy is that for a given luminosity (for instance in stages during the second crossing) models intrinsically possess a lower mass. This is indeed characteristic of models with overshoot. In fact, at any given initial mass, the tracks cross the instability strip at higher luminosity than classical models, or conversely, at any given luminosity the correspondent Cepheid mass is significantly lower (Matraka et al. 1982; Bertelli et al. 1985). To illustrate the point, in Figure 5 we plot the luminosity versus $M_{p u l}$ using the data compiled by Cox (1980) together with the theoretical mass-luminosity relation for classical models (Becker et al. 1977) and models with overshoot [see the relations (13a) and (13b) below]. Although the scatter is large, it can be seen that classical models hardly match the bulk of data, because most of the stars appear to be either too luminous for their masses or too light for their luminosity (pulsational masses grossly in error?) or the classical relationship has to be shifted toward lower masses. It goes without saying that both coordinates are highly uncertain as indicated by the shifts that two prototype Cepheids, U Sgr and S Nor, would suffer when Pel's (1985) data are adopted. Amazingly enough, after correction these two stars fall along the mass-luminosity relationship for models with overshoot. Shifts of the same size are likely to be expected for all stars in the sample. The conclusion from all this can only be that the solution of the mass discrepancy requires improvements on both the observational and theoretical side, even though the net advantage offered by models with overshoot ought to be borne in mind. 
4. THE FREQUENCY-PERIOD DISTRIBUTION

It is clear that in a statistically large sample of stars the intersection of the instability strip with the blue band of core He-burning models will give rise to a population of Cepheids whose periods distribute according to a specific law that is easy to understand. The period distributions, number of stars per period bin, $\mathrm{N}(\log \mathrm{P})$, observed in the solar vicinity, Milky Way, M31, SMC, and IMC and shown in Figure 6 (Becker et al. 1977), are characterized by three parameters, the short period cutoff $P_{0}$, the period $P_{1}$ at the maximum of the distribution, and the rate at which Cepheids with $\mathrm{P}>\mathrm{P}_{1}$ decay in number with respect to the period. It is soon evident that the $\mathrm{N}(\log \mathrm{P})$ distribution varies from one system to another both in overall shape and the characteristic periods $P_{0}$ and $P_{1}$. The underlying reasons for these variations can be understood in a simple way.

Following Becker et al. (1977), Iben \& Tuggle (1975) and Serrano (1983), we start supposing that all Cepheids possess the same chemical composition (indeed this is not the case), which implies that only one blue band of core He-burning models and one instability strip (both are known to depend on the chemical composition) can be used. The

Fig. 5. Luminosity versus mass relationship for Cepheid stars. The full dots show the luminosities and pulsational masses of Cox (1980). The arrows indicate the revision made by Pel (1985) for U Sgr and S Nor. Standard models of Becker et al (1977), and those with convective overshoot are shown.

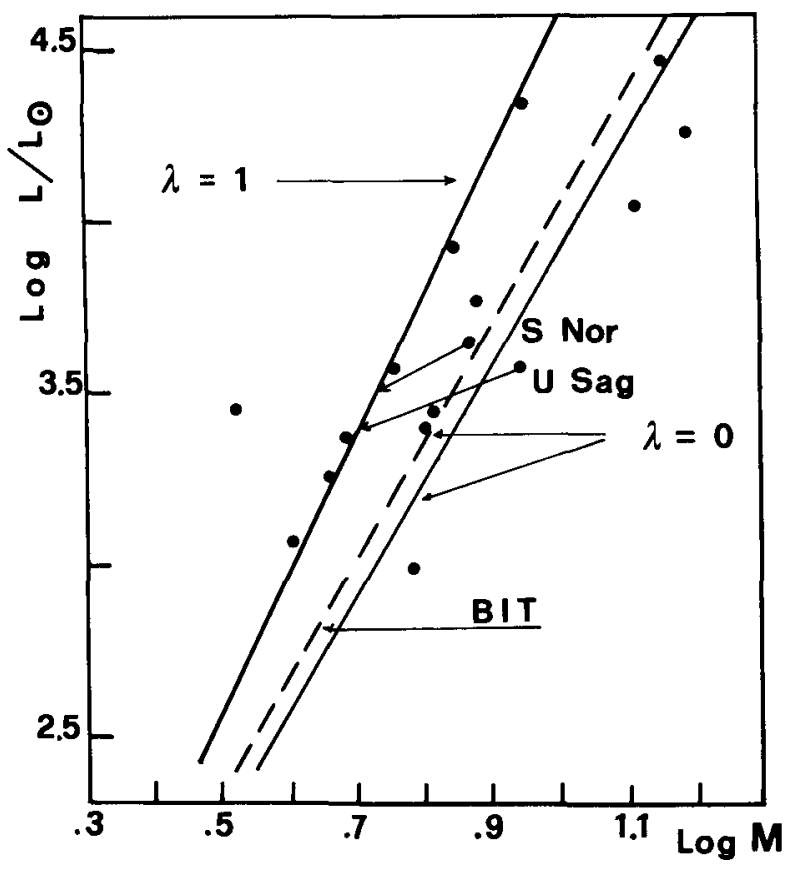


intersection of the two bands define two characteristic luminosities $\mathrm{L}_{0}$ and $I_{\max }$. $I_{0}$ corresponds to the luminosity of the model (mass) whose blue loop is extended enough to reach the red edge of the instability strip, whereas $L_{\max }$ is the last model (mass), above which the blue loop

Figure 6. The frequency-period distributions, $N(\log P)$, defined for Cepheid stars in the Milky Way, M31, SMC and IMC are shown. In each panel the total number of stars in the sample is given. The low period cutoff $\mathrm{P}_{0}$ and the period $\mathrm{P}_{1}$ at the maximum of the distribution are indicated by the vertical arrows. This figure is adapted from Becker et al. (1977).

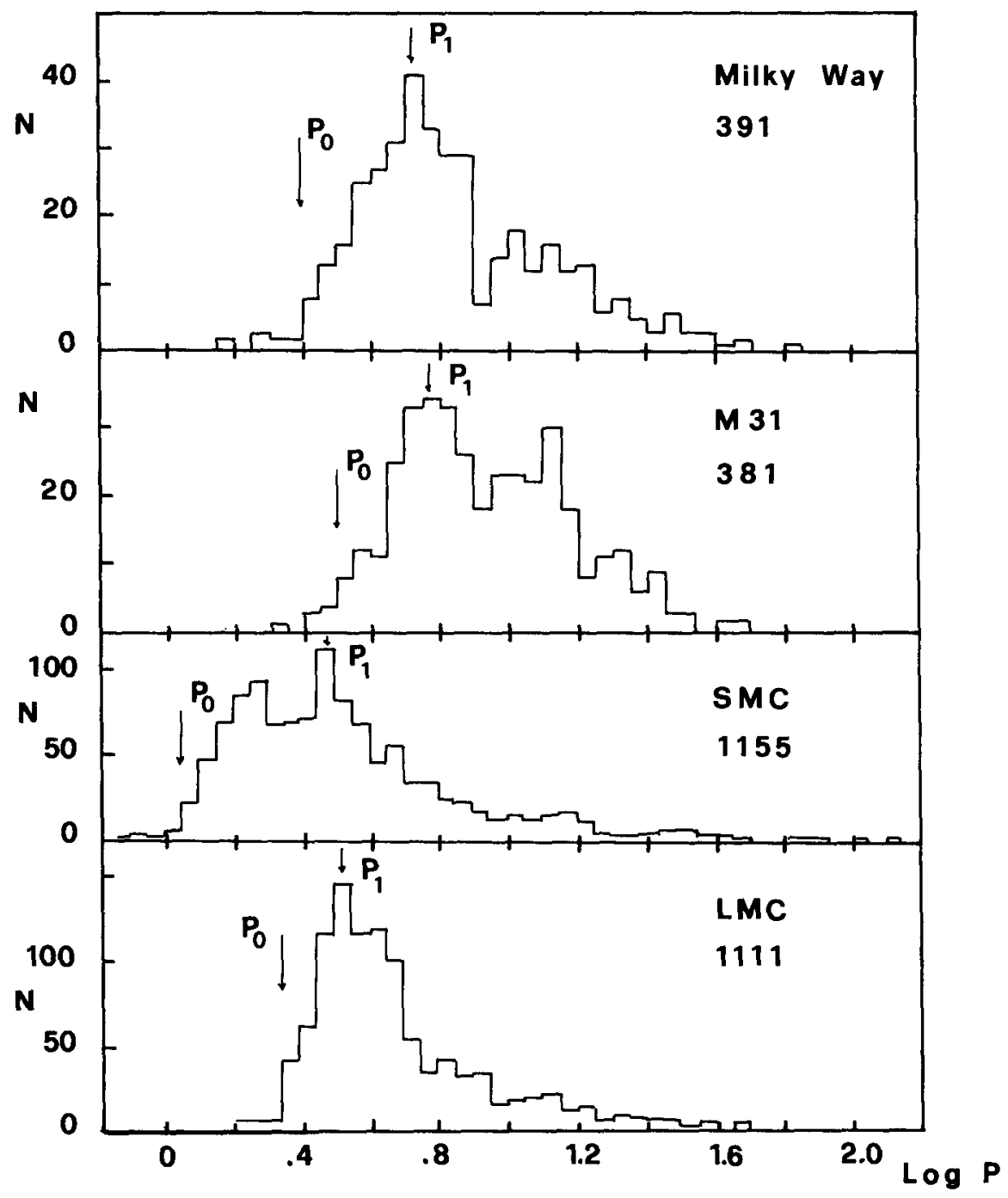


extends so far into the blue that the instability strip cannot be crosssed on nuclear time scales. $L_{\max }$ is determined by the intersection of the blue edge of the instability with the blue band. It goes without saying that a star (mass) would exist whose blue loop has the appropriate extension to spend all the blue He-burning phase within the instability strip. The luminosity of this particular models is indicated by $L_{1}$. Let $\boldsymbol{w}(t)$ be the total stellar birthrate (in some units dictated by the observational sample of Cepheids) and $\Phi(M)$ the initial mass function assumed to be independent of time and chemical composition. Then $\boldsymbol{\Psi}\left(t^{\prime}\right) \Phi(M) d M d t^{\prime}$ is the number of stars born between $t^{\prime}$ and $t^{\prime}+d t^{\prime}$ and mass between $M$ and $M+d M$. Hence the number of Cepheids with mass between $M$ and $M+d M$ is given by

$$
\mathrm{dN}=\boldsymbol{\Psi}\left(t-T_{M}\right) \Phi(M) \Delta t(M) d M
$$

where $T_{M}$ is the lifetime of a star of mass $M$ from ZAMS to the edge of the instability strip and $\Delta t(M)$ is the crossing time of the instability strip for this stellar mass. Since for all relevant masses $T_{M}$ is short compared to the typical time scales of star formation, we may write

$$
\mathrm{dN} / \mathrm{d} \log \mathrm{P}=\boldsymbol{\Psi}(\mathrm{t}) \mathrm{M \Phi}(\mathrm{M}) \Delta \mathrm{t}(\mathrm{M}) \ln 10 \mathrm{~d}(\log \mathrm{M}) / \mathrm{d}(\log \mathrm{P}) .
$$

The incorporation of the pulsation theory starts from the general relationship between $\mathrm{P}, \mathrm{L}, \mathrm{T}_{\text {eff }}$ and $\mathrm{M}$ for a Cepheid pulsating in the fundamental mode anywhere in the instability region given by Iben \& Tuggle (1975), and the period-luminosity relationship of Becker et al. (1977) for models in the second transit, which to a first approximation is nearly independent of composition,

$$
\log L=2.51+1.25 \log \mathbf{P} \text {, }
$$

where $\mathrm{L}$ is in solar units and $\mathrm{P}$ in days.

Finally, with the aid of a mass-luminosity relationship we may determine $d(\log M) / d(\log P)$. The mass-luminosity relation for models in the blue band of the core He-burning depends on the underlying evolutionary scenario and it is very sensitive to chemical composition, opacity, nuclear reaction rates, mass loss, mixing mechanisms, and finally mass range. In the following we will use the following relations which based on models with convective overshoot, the new rate for the ${ }^{12} \mathrm{C}(\boldsymbol{\alpha}, \mathbf{y}){ }^{16} \mathrm{O}$, and no mass loss by stellar wind:

$$
\log \mathrm{L}=0.924+3.61 \log \mathrm{M},
$$

for $Z=0.020$ and $4<M<9 M_{\odot}$ and

$$
\log L=1.511+3.22 \log M,
$$

for $\mathrm{Z}=0.001,2<\mathrm{M}<9 \mathrm{M}_{\odot}$, where $\mathrm{L}$ and $\mathrm{M}$ are in solar units. Similar relations for classical models can be found in Becker et al. (1977) or constructed from the models of Becker (1981). The instability strip is 
assumed to have a constant width in effective temperature $W=\log \left(T_{\text {eff }}\right)_{B E}-\log \left(T_{\text {eff }}\right)_{R E}$, where $B E$ and $R E$ stand for blue and red edge, respectively. The width $W$ is however very uncertain, ranging from the theoretical value of 0.04 (Becker et al. 1977) to the more recent determination of 0.15 (Pel \& Lub, 1978; Dupree, 1980).

The $\Delta t(M)$ relation must be derived from theoretical models. Like the mass-luminosity relationship, it depends on the underlying evolutionary scenario and details of the input physics of model calculations. In the following we adopted the values derived from models with convective overshoot. Finally, the Salpeter law with slope $x=2.35$ is adopted in the $\mathrm{N}(\log \mathrm{P})$ distribution presented below.

Although the study of Becker et al. (1977) clearly shows the potential capability of the theory to reproduce the observed $N(\log P)$ distribution in seven galaxies of the Local Group, when a detailed comparison is made the agreement is rather poor. In fact, they find that the short period cutoff, $\mathrm{P}_{0}$, and the maximum peak, $\mathrm{P}_{\mathbf{1}}$, in the distribution occur at progressively larger values of $\log p$ when galaxies are ordered according to their heavy element abundance. However, on one hand their $\mathrm{P}_{0}{ }^{\prime} \mathrm{s}$ and $\mathrm{P}_{1}$ 's are too low compared to the observational values, on the other hand to explain the excess of long period Cepheids, they had to adopt a two component birthrate. The secondary component (that of long period, hence massive stars) would have an amplitude 5 to 50 times greater than the primary component. Serrano (1983) re-examining this problem concludes that the excess of long period Cepheids in the galactic $N(\log P)$ distribution may be explained by a gradient of star formation combined with a gradient in metallicity. The observational data for the solar vicinity and the two Magellanic clouds together with the predictions from classical models are summarized in Table 2. The metallicity assigned to each galaxy is a mean value of current estimates in literature. Regardless of the excess of long period Cepheids, the disagreement between observational $\mathrm{P}_{0}^{\prime} \mathrm{s}$ and $\mathrm{P}_{1}^{\prime} \mathrm{s}$ and theoretical predictions is hard to reconcile on the basis of classical models. Models with overshoot can alleviate the discrepancy since they predict longer $\mathrm{P}_{0}{ }^{\prime} \mathrm{s}$. This can be easily understood looking at the partial derivatives of $\mathrm{P}$ with respect to $\mathrm{L}, \mathrm{T}_{\mathrm{eff}}, \mathrm{M}$ in the $\mathrm{P}-\mathrm{L}-\mathrm{T}_{\text {eff }}-\mathrm{M}$ relationship of Iben \& Tuggle (1975), where the period increases at increasing luminosity, whereas it decreases at decreasing effective temperature and mass. For any given mass, models with overshoot run at higher luminosity and lower effective temperature. However, even these latter models predict high $\mathrm{P}_{0}$ 's. To date, two ways out have been suggested to increase $\log \mathrm{P}_{1} / \mathrm{P}_{0}$, namely: i) a much wider instability strip; ii) an increase in the slope of the blue band, or in other words a blue band not extending too much to high effective temperatures. The first possibility has been already considered by Bertelli et al. (1985), but it turned out to be insufficient unless $W$ gets very large. A blue band of core He-burning models more vertical in the HR diagram and closer to the Hayashi limit can be obtained under favourable circumstances (higher opacity and metallicity, more efficient mass loss, etc.). Nevertheless, it can be easily understood that both $W$ and the slope of the blue band affect $P_{1}$ more than $P_{0}$. Since models with 
overshoot and metallicity appropriate for the solar vicinity almost matched $P_{1}$, what is really needed is to decrease $P_{0}$, hence include stars of lower mass and at the same time to obey the constraint of $P_{0}$ and $P_{1}$ being increasing toward the galactic centre, where the metallicity is higher than in the solar neighborhood. This is easily achieved by allowing for a dispersion in chemical composition as already suggested by Becker et al. (1977). We find that adopting a gaussian dispersion in metallicity with $\sigma_{z}=0.0015$ both the periods $\mathrm{P}_{0}$ and $\mathrm{P}_{1}$ for the solar vicinity can be matched and the $\mathrm{N}(\log \mathrm{P})$ distribution observed in the Milky Way, LMC and SMC can be accounted for. The results for three galaxies are given in Table 3 . In Figure 7 we show the $\mathrm{N}(\log \mathrm{P})$ distribution expected for the LMC together with its observational counterpart.

Finally, we would like to point out that both the new models and the (small) dispersion in metallicity concur to offer an explanation of the $\mathrm{N}(\log \mathrm{P})$ distribution without invoking a two component birthrate. In fact, in models with overshoot the blue band of core He-burning tends to move back toward the Hayashi line at relatively higher masses

$\left(M>9 M_{\odot}\right)$, the tendency being enhanced by increasing the metallicity and/or the opacity (see below). This would allow for a wider range of masses spending a large fraction, if not all, of the blue band lifetime within the instability strip. Another interesting consequence of the

Table $2(*)$

\begin{tabular}{|c|c|c|c|c|c|c|}
\hline$\overline{\mathrm{Y}}$ & $\bar{Z}$ & $\lambda$ & $\log \overline{\mathrm{P}}_{1}$ & $\overline{\log } \overline{\mathrm{P}}_{0}$ & $\bar{M}_{\min }$ & Notes \\
\hline & $\begin{array}{r}0.020 \\
<0.020 \\
0.009 \\
0.002\end{array}$ & & $\begin{array}{l}0.90 \\
0.75 \\
0.52 \\
0.48\end{array}$ & $\begin{array}{l}0 . \overline{30} \\
0.30 \\
0.20 \\
0.10\end{array}$ & & $\begin{array}{l}\text { Solar Vicinity } \\
\text { Milky Way } \\
\text { LMC } \\
\text { SMC }\end{array}$ \\
\hline $\begin{array}{l}\overline{0.280} \\
0.280 \\
0.280 \\
0.280 \\
0.360\end{array}$ & $\begin{array}{l}0.001 \\
0.010 \\
0.030 \\
0.020 \\
0.020\end{array}$ & & $\begin{array}{l}\text { Classica } \\
-0.28 \\
-0.38 \\
-0.73 \\
-0.08 \\
0.68\end{array}$ & $\begin{array}{c}\text { Models } \\
<-\overline{0.33} \\
-0.61 \\
-0.81 \\
-0.26 \\
0.60\end{array}$ & & (a) \\
\hline $\begin{array}{l}0.280 \\
0.280 \\
0.280 \\
0.299\end{array}$ & $\begin{array}{l}0.020 \\
0.020 \\
0.020 \\
0.001\end{array}$ & $\begin{array}{l}1 \\
0 \\
0.5 \\
1.0 \\
1.0\end{array}$ & $\begin{array}{l}\text { els with } \\
0.45 \\
0.85 \\
1.02 \\
0.06\end{array}$ & $\begin{array}{c}\text { overshoot } \\
0.37 \\
0.77 \\
0.94 \\
-0.02\end{array}$ & $\begin{array}{l}5.46 \\
2.05\end{array}$ & (b) \\
\hline
\end{tabular}

(*) Width of the instability strip $\log \mathrm{T}_{\text {eff }}=0.04$

(a) Becker et al. (1977)

(b) Bertelli et al. (1985) 
fact that the blue core He-burning band of models with overshoot, after a maximum diplacement toward the blue, moves back to the red giant region is that it may naturally lead either to a broad $N(\log P)$ distribution or even to a bimodal distribution of periods, which is not due to inhomogeneities in the initial chemical composition but it is intrinsic to stellar models. As a matter of fact, the distributions observed in SMC, M31 and perhaps the Milky Way seem to suggest a bimodal $N(\log P)$ distribution. However, whether or not those observational distributions are really bimodal cannot be easily assessed at the present time, and therefore this point is left to future investigation.

5. EVOLUTION WITH VARIED RADIATIVE OPACITY

Since the early studies of Henyey et al. (1965), Fricke et al. (1971), Robertson (1972), Johnson \& Whittaker (1975), Carson \& Stothers (1976), to name a few, it has been known that changing the opacity used either in stellar interiors or in stellar atmospheres can

Table 3

\begin{tabular}{lllll}
\hline Site & $\mathrm{Z}$ & $\mathrm{Z}$ & $\log \mathrm{P}$ & $\log \mathrm{P}$ \\
\hline Milky Way & 0.020 & 0.006 & 0.90 & 0.30 \\
LMC & 0.009 & 0.007 & 0.52 & 0.37 \\
SMC & 0.002 & 0.001 & 0.48 & 0.28 \\
\hline
\end{tabular}

Figure 7. The theoretical N(logP) distribution from models with overshoot and dispersion in metallicity (thin dashed line). This is compared with the $N(\log P)$ defined for the LMC (thick line).

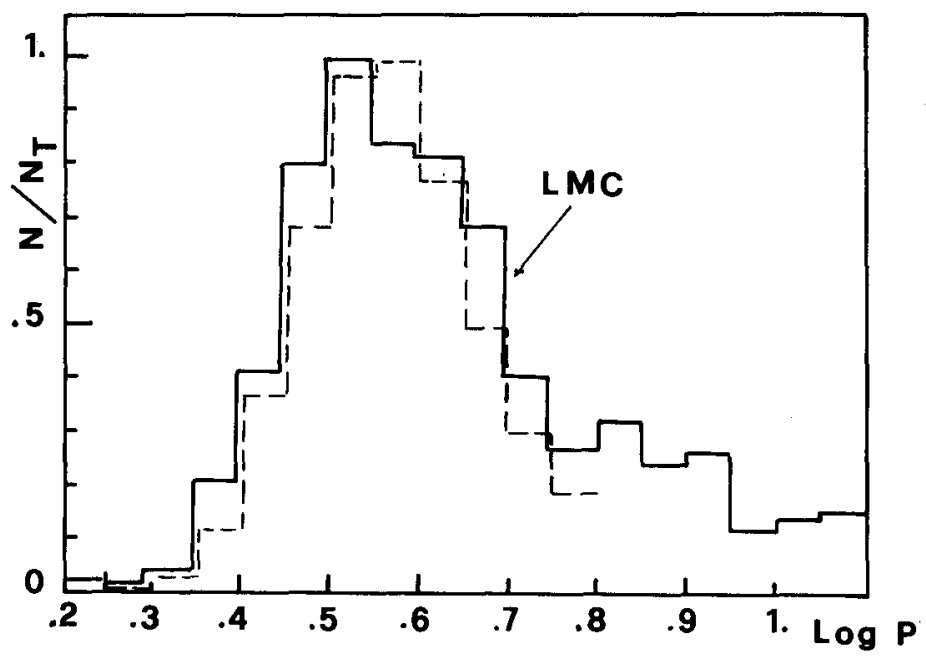


have a great impact on a star's evolutionary history and path in the HR diagram. Almost any property of the star's structure depends on opacity including luminosities, lifetimes, occurrence of semiconvection, extension of the blue loops, etc. With regard to Cepheids, the most important question addressed in the past was whether the Carson (1976) or the Los Alamos opacities (Cox \& Stewart 1965, 1970) are to be used (see Carson \& Stothers, 1976; Cox \& Tabor, 1976, respectively). The primary difference in behaviour between the two opacities is that Carson's opacity has a larger contribution from He and (mostly) CNO elements in the temperature range $6.5<\log T<4.3$ and moderate densities $\left(10^{-4}\right.$ to $\left.10^{-5} \mathrm{gr} \cdot \mathrm{cm}^{-3}\right)$. Since to date the controversy has not been settled from the standpoint of the fundamental physics going into the calculation of radiative opacities, the question has been addressed from an empirical point of view, and at least two arguments have been advocated to suggest an increase in the heavy element opacity, in particular that due to CNO elements. These are the relative numbers of supergiant stars per spectral type (Bertelli et al. 1984) and the mass anomaly and period ratios of bump Cepheids (Simon, 1982). They are briefly summarized below.

Supergiant stars. The effects of the Carson opacity on models of massive stars have been investigated by Stothers (1976), and Stothers \& Chin $(1977,1978)$. The most interesting results of those calculations were that the locus of the zero age main sequence progressively shifted toward cooler effective temperature, and the main sequence band systematically broadened by increasing the metallicity, decreasing the mixing length parameter used to describe the outermost convective zone, and increasing the mass of the star. For a suitable combination of the three parameters the main sequence band may even extend across the whole HR diagram. Though interesting, Carson's opacities and, in turn, the models for massive stars based on their usage never received a general consensus. Among others, the main objection resided in the predicted location of the zero age main sequence which does not agree with the location of luminous early type stars obtained from the so-called hot temperature scale, transferring spectral types into effective temperature and bolometric corrections, currently in use. The question was subsequently reopened by Bertelli et al. (1984), who starting from the suggestion by Simon (1982), to be discussed below, and the criticism of Iben \& Renzini (1984) on current radiative opacities in general, proposed a modification to standard radiative opacities, mimicking the bump in the Carson opacity, to reconcile the observational frequency of luminous $O B$ type and supergiant stars with theoretical expectation. Star counts in fact indicate that a large fraction (up to $40 \frac{\mathrm{g}}{8}$ ) of stars fall outside the region of core H-burning (even allowing for mass loss by stellar winds and convective overshoot), whereas the theoretical expectation is 10 to $20 \%$. It appears that either star counts are still severely biased by incompleteness and/or selection effects or the theoretical main sequence band ought to extend at least up to spectral type A0 (see Chiosi \& Maeder 1986 for a thorough discussion of this point). The following modification to the opacity. was proposed by Bertelli et al. (1984): 


$$
K=K_{\text {CS }}\left(1+X \exp \left[-10(5.8-\log T)^{4}\right]\right)
$$

where $K_{C s}$ stands for the Cox and Stewart opacity, and $X$ is an adjustable parameter. As Carson's opacities show the bump in the range of temperature $5.4<\log T<6.2$, and are higher at lower densities, the bell-shaped bump given by relation (14) was centered at $\log T=5.8$, whereas no dependence on density was considered. Bertelli et al. (1984) conclude that models characterized by mass loss at a moderate rate, overshoot from the convective core, and opacity of the CNO ionization layers slightly enhanced with respect to the standard one $(X=2)$ can easily account for a much wider main sequence band and for the star counts.

Cepheid stars. The mass anomalies and period ratios encountered by double-mode (admixture of both fundamental and first overtone pulsations) and "bump" Cepheids (the bump often appears in the light curves of Cepheids with periods in the range 7-11 days), which cannot be accounted for by classical models, led Simon (1982) to suggest that an increase by a factor of 2 to 3 in the heavy element opacity could help to remove the mass anomaly.

However, Simon's (1982) and Bertelli's et al. (1984) suggestions have been questioned by Magee et al. (1984), who claim that such a large increase in opacity is incompatible with atomic physics. An opposite conclusion has been reached by Iglesias et al. (1987), who argue that an improved treatment of the atomic physics can significantly increase the Rosseland mean opacities of metals in astrophysical mixtures. This possibility has been recently reinforced by Andreasen (1988), who claims that enhanced metal opacity is needed to reduce the Cepheid period ratio discrepancies. The above contrasting conclusions simply indicate that the topic is still a matter of vivid debate, and therefore it is not implausible that the true radiative opacities will eventually turn out to be larger than usually accepted.

In order to clarify the role played by variations in the contribution of heavy elements to the radiative opacity, we may use the following relation proposed by Renzini (1984b) and adopted by Bertelli \& Bressan (1988) in the calculations of stellar models we are going to present,

$$
K=K_{X Y}+A \Delta Z \sigma\left[1+X f(\rho, T) \exp \left\{-4 \alpha \log \left(T / T_{0}\right)\right\}\right],
$$

where $K_{X Y}$ is the opacity of a metal-free mixture of hydrogen and helium. The term $A \Delta Z$ gives the contribution of heavy elements to the classical radiative opacity. This term is evaluated, at each value of $\rho$ and $T$, subtracting the opacity of a metal-free mixture with given $X$ and $Y$ from the opacity of a mixture having the same $X$ and $Y$ but a metal abundance $z$. $\sigma$ is a parameter by which the contribution of heavy elements can be varied all over the domain of temperatures and densities. Furthermore, $f(p, T)$ is a suitable function defining in the $\rho-T$ plane a band along which the opacity enhancement, given by the exponential term, is allowed to occur, mimicking the opacity bump proposed by Bertelli et al. (1984). $\mathrm{T}_{0}$ is the central value of the temperature interval relative to the 
ultimate ionization of the CNO group of elements $\left(T_{0}=10^{6} \mathrm{~K}\right)$. The requirement of matching the opacity increase proposed by Simon (1982) and Bertelli et al. (1984) allows us to determine $\boldsymbol{a}, f(\boldsymbol{p}, T)$ and $X$.

Effects of a bump in the opacity. Assuming $\sigma=1$ and $X=2$ to 3 , relation $(1 \overline{5})$ simulates a bump in the opacity centered on the temperature $T_{0}$ and suitably modulated in density by means of $f(\rho, T)$. The effects of a bump on the properties of massive stars have been already described above. They do not depend on whether or not convective overshoot is allowed to occur. The behaviour of intermediate mass stars is more complicated. The core H-burning phase of classical models is not affected to any appreciable extent by a bump in the opacity, whereas the core He-burning phase is more sensitive to it. In fact the calculations show that either very extended loops may occur (Robertson 1972) or that very minor variations in the luminosity and effective temperature can be seen (Becker 1985). On the contrary, core He-burning models with overshoot respond very much to this type of enhancement in the opacity (even of modest size). Almost independently of the efficiency of convective overshoot, hence increase in the mass of the convective core, the loops are strongly inhibited for all masses greater than about $5 \mathrm{M}_{\odot}$ when the metallicity is about solar $(\mathrm{z}=0.02)$. At decreasing metallicity, the loops develop again though much narrower at increasing mass. The luminosity of these models is about the same as in models with overshoot and standard opacity. As a consequence of this, the C-shaped blue band of core He-burning models, that was already seen to occur in models with overshoot and standard opacity, is now found to be extremely sensitive to the opacity enhancement and, for given values of $X$ and $T_{0}$, to the metallicity. The apex of this band, which was very blue and luminous in classical models without convective overshoot (see Figure 1), and which became less luminous and much redder in models with overshoot and standard opacity (see Figure 2), may now occur at even fainter luminosities and cooler effective temperatures, depending on the opacity enhancement and metallicity of the star. If these numerical experiments are confirmed by detailed opacity calculations, the impact on the properties of Cepheid stars and, in particular, on the $\mathrm{N}(\log \mathrm{P})$ distribution is of paramount importance. It goes without saying that for appropriate metallicities (within the range of plausible values) the instability strip is either easily crossed twice on nuclear time scales or intersected by the blue band over a range of luminosities (masses) much wider than ever before. Although such a possibility is already offered by models with overshoot but standard opacities, provided that the right combination of convective overshoot and metallicity is adopted (in general, substantial overshoot and/or very low metallicities were needed), now due to the varied opacity it may occur for "normal" overshoot and metallicities. Therefore the changes advocated by Simon (1982) are potentially permissible. We like to speculate that the bimodality in the $\mathrm{N}(\log \mathrm{P})$ distribution perhaps seen in the Milky Way, SMC and M31 could be the result of this variation in the opacity. 
Effects of an overall increase in the heavy element opacity. This can be simulated by assuming in relation (15) that $X=0$ and $\sigma \neq 0$ (say 2 or 3). This type of variation would have very severe consequences. In brief, the luminosity and effective temperature of the models both in core $\mathrm{H}-$ and He-burning phases are expected to decrease, the lifetimes to increase, and loops to be almost always suppressed unless unacceptably low metallicities are assumed. Models of this type have been calculated by Nasi \& Forieri (1988) in the range of massive stars (incorporating mass loss and convective overshoot) and used to simulate the observational distribution of luminous stars in the colour magnitude diagram. In this mass range, the effects of this type of opacity are marginal and indeed the models have been found to match reasonably well the observational data. On the contrary, no systematic analysis of the effects given by this type of opacity variation on the properties of intermediate and low mass stars has been made.

\section{SURFACE COMPOSITION OF CEPHEIDS}

With few rare exceptions, the vast majority of Cepheid stars have undergone the first dredge-up while ascending the RGB, or in other words external convection penetrates the inner layers that in the past experienced nuclear processing with consequent changes of the surface abundances. Classical calculations of stellar models (see Becker \& Iben, 1979) predict that, as a consequence of the dredge-up, the surface abundance of ${ }^{14} \mathrm{~N}$ should increase by a factor of two to three and the abundance of ${ }^{16} \mathrm{C}$ should decrease by $30 \%$ to 408 (see also Iben \& Renzini, 1984). The surface abundances of Cepheid stars are therefore particularly interesting as they can be used as a probe of the interior structure and efficiency of convective mixing. In relation to this we recall that: i) Cepheids have main sequence progenitors typically rotating fast enough that enhancement in mixing and abundance variations at the surface are likely to be expected; $i i)$ classical models, for which the above abundance variations have been calculated, can now be replaced by models with convective overshoot, which by virtue of their larger internal mixed and nuclear processed regions can more easily show abundance variations at the surface. Luck \& Lambert (1981) were the first to measure the surface CNO abundances of Cepheids with the conclusion that the distribution of CNO elements indicates that mixing of matter in which considerable amounts of 0 (as well C) have been processed into $N$ (so-called $O N$ processing) far in excess of the classical prediction. Becker \& Cox (1982) discussed the implications of how stellar models would be altered to account for these observations. However, Luck and Lambert's result was questioned by Iben \& Renzini (1984), who rediscussed the data and came to the conclusion that there is no evidence that ON-cycled material has been exposed to the surface, while there is some evidence that $\mathrm{CN}$ materials are present, however in agreement with the canonical predictions for the first dredge-up. Models incorporating convective overshoot have not yet been analysed from the standpoint of surface abundances. 
Finally, we would like to conclude this section, touching upon another crucial problem related to the chemical composition of Cepheid stars, namely the dependence of the period-luminosity (PL) and period-luminosity-colour (PLC) relations on the helium and metal abundances. These relations in fact play an important role in establishing the extragalactic distance scale. This problem has been addressed by Stothers (1988), who has derived PL and PLC relations using relevant data from stellar evolution, atmosphere, and pulsation theory incorporating the composition dependences for all quantities. As expected, the PLC relation in $B$ and $V$ magnitudes is much more sensitive to chemical composition than is the PL relation. The new calibrations are used to derive the distance moduli of the Magellanic Clouds. These corrected for the abundance effects are $(\mathrm{m}-\mathrm{M})_{0}=18.51+0.06$ for $\mathrm{LMC}$ and $(\mathrm{m}-\mathrm{M})_{0}=18.8+0.06$ for SMC.

\section{ACKNOWLEDGEMENTS}

This work has been financed by the National Group of Astronomy (GNA) and the Italian Space Research Program (PSN) of the National Council of Research of Italy (CNR) .

\section{REFERENCES}

Andreasen, G. K. (1988). Astron. \& Astrophys., 201, 72.

Barbaro, G. \& Pigatto, L. (1984). Astron. \& Astrophys., 136, 355.

Becker, S.A. (1981). Ap.J.Suppl., 45, 478.

Becker, S.A. (1985). In Cepheids: Theory and Observations, ed. B.F. Madore, p. 104 Cambridge: Cambridge University Press.

Becker, S.A. \& Cox, A.N. (1982). Ap.J.,260, 707.

Becker, S.A. \& Iben, I. (1979). Ap.J., 232, 831.

Becker, S.A., Iben, I. \& Tuggle, R.S. (1977). Ap.J., 218, 633.

Becker, S.A. \& Mathews, G.J. (1983). Ap.J.,270, 155.

Bergh, S. van den, (1981). Astron. \& Astrophys. Suppl.,46, 79.

Bertelli, G., Bressan, A. \& Chiosi, C. (1985). Astron. \& Astrophys., 130, 279.

Bertelli, G., Bressan, A. \& Chiosi, C. (1985). Astron. \& Astrophys.,150, 33.

Bertelli, G., Bressan, A., Chiosi, C. \& Angerer, K. (1986a). Astron. \& Astrophys.Supp1.,66, 191.

Bertelli, G., Bressan, A., Chiosi, C. \& Angerer, K. (1986b). In The Age of Star Clusters, ed. F. Caputo, Mem.Soc.Astron.It $157,427$.

Blanco, B.M., Blanco, V.M. \& McCarthy, M.F. (1978). Nature, 271, $\overline{63} 8$.

Blanco, B.M., McCarthy, M.F. \& Blanco, V.M. (1980). Ap.J., 2 $\overline{42}, 948$.

Böhm-Vitense, E. (1986). Ap.J., 303, 262.

Boothroyd, A.I. \& Sackmann, I.J. (1988a). Ap.J., 328, 632.

Boothroyd, A.I. \& Sackmann, I.J. (1988b). Ap.J., $328,641$.

Boothroyd, A.I. \& Sackmann, I.J. (1988c). Ap.J., $\overline{328}, 653$.

Boothroyd, A.I. \& Sackmann, I.J. (1988d). Ap.J., $\underline{328}, 671$.

Bressan, A. \& Bertelli, G. (1988). in preparation.

Bressan, A., Bertelli, G. \& Chiosi, C. (1981). Astron. \& Astrophys.,102, 25. 
Bressan, A., Bertelli, G. \& Chiosi, C. (1986). In The Age of Star Clusters, ed. F. Caputo, Mem.Soc.Astron.It.,57, 411.

Brunish, W.M. \& Willson, L.A. (1987). In Stellar Pulsation, eds. A.N. Cox, W.M. Sparks \& S. G. Starrfield, p. 27. Berlin: Springer-Verlag.

Buonanno, R., Corsi, C.E. \& Fusi-Pecci, F. (1985). Astron. \& Astrophys. , 145, 97.

Carson, T.R. (1976). Ann.Rev.Astron. Astrophys.,14, 95.

Carson, T.R. \& Stothers, R. (1976). Ap.J.,204, $\overline{46} 1$.

Castellani, V., Chieffi, A.. Pulone, L. \& Tornambe, A. (1985). Ap.J., 296, 204.

Chiosi, C. (1986). In Nucleosynthesis and Stellar Evolution, 16th Saas-Fee Course, eds. B. Hauck et al., p. 199. Geneva: Geneva Observatory.

Chiosi, C., Bertelli, G. \& Bressan, A. (1987). In Late Stages of Stellar Evolution, eds. S. Kwok \& S.R. Pott $\overline{a s c h, ~ p . ~ 239 . ~ D o r d r e c h t: ~}$ Reidel.

Chiosi, C., Bertelli, G., Meylan, G. \& Ortolani, S. (1988). Astron.\& Astrophys., submitted.

Chiosi, C. Maeder, A. (1986). Ann.Rev.Astron.Astrophys., 24, 329.

Cox, A.N. (1980). Ann. Rev.Astron. Ast rophys.,18, 15.

Cox, A.N. (1985). In Cepheids: Theory and Observations, ed. B.F. Madore, p. 126. Cambridge: Cambridge University Press.

Cox, A.N., Deupree, R.G., King, D.S. \& Hodson, S.W. (1977). Ap.J.,214, L127.

Cox, A.N. \& Stewart, J.N. (1965). Ap.J.Suppl.,11, 22.

Cox, A.N. \& Stewart, J.N. (1970). Ap.J.Suppl., $\overline{19}, 243$.

Cox, A.N. \& Tabor, J.E. (1976). Ap.J.Suppl., 31, 271.

Cox, J.P. (1980). Theory of Stellar Pulsation. Princeton, New Jersey: Princeton University Press.

Deupree, R.G. (1977). Ap.J., 211, 509.

Deupree, R.G. (1980). Ap.J., $\overline{236}, 225$.

Endal, A.S. \& Sofia, S. (1976). Ap.J.,210, 184.

Endal, A.S. \& Sofia, S. (1978). Ap.J., $220,290$.

Endal, A.S. \& Sofia, S. (1979). Ap.J., $\overline{232}, 531$.

Fowler, W.A. (1984). Rev.Mod.Phys.,56, 149.

Fowler, W.A., Caughlan, G.R. \& Zimmerman, B.A. (1967). Ann. Rev. Astron. Astrophys., 5, 525.

Fowler, W.A., Caughlan, G.R. \& Zimmerman, B.A. (1975). Ann. Rev. Astron. Astrophys. $13,69$.

Fricke, K., Stobie, R.S. \& Strittmatter, P.A. (1971). M.N.R.A.S.,154, 23.

Hanson, R.B. (1979). In The HR Diagram, eds. A.G.D. Philip \& D.S. Hayes, p. 154. Dordrecht: Reidel.

Harris, M.J., Fowler, W.A., Caughlan, G.R. \& Zimmerman, B.A. (1983) . Ann. Rev. Astron. Ast rophys. , 21, 165.

Heckman, T.M. (1976). Ap.J.Suppl., 36, $\overline{451}$.

Henyey, L.G., Vardja, M.S. \& Bodenheimer, P. (1965). Ap.J.,142, 841. Hollowell, D.E. (1987). In Late Stages of Stellar Evolution, eds. S. Kwok \& S. R. Pottasch, p. 239. Dordrecht: Reidel. 
Hollowell, D.E. (1988). Ph. D. Thesis, University of Illinois. Iben, I. (1972). Ap.J.,178, 433.

Iben, I. (1974). Ann. Rev.Astron. Astrophys., 12, 215.

Iben, I. (1975a). Ap.J.,196, 525.

Iben, I. (1975b). Ap.J., 196, 549.

Iben, I. (1976). Ap.J., $2 \overline{08}, 165$.

Iben, I. (1981). Ap.J., $\overline{246}, 278$.

Iben, I. \& Renzini, A. (1983). Ann.Rev.Astron.Astrophys., 21, 271.

Iben, I. \& Renzini, A. (1984). Physics Reports 105, no. 6, 329.

Iben, I. \& Tuggle, R.S. (1972a). Ap.J.,173, 135.

Iben, I. \& Tuggle, R.S. (1972b). Ap.J., $\overline{178}, 441$.

Iben, I. \& Tuggle, R.S. (1975). Ap.J.,197, 39.

Iglesias, C.A., Rogers, F.J. \& Wilson, B.G. (1984). Ap.J.,322, L45.

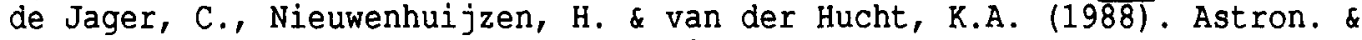
Astrophys. Suppl.,72, 259.

Johnson, H.R. \& Whittaker, R.W. (1975). M.N.R.A.S.,173, 523.

Kettner, K.U., Becker, H.W., Buchmann, L., Görres, J., Kräwinkel, H., Rolfs, C., Schmalbrock, P., Trautvelter, H.P. \& Vlieks, A. (1982). Z.Phys.A-Atoms and Nuclei, 308, 73.

Kippenhahn R., Meyer-Hofmeister, E. \& Weigert, A. (1970). Astron. \& Astrophys., $5,155$.

Kraft, R.P. (1970). In Spectroscopic Astrophysics, ed. G.H. Herbig, p. 385. Berkeley: University of California Press.

Lamers, H.J.G.L.M. (1981). Ap.J., 245, 593.

Lattanzio, J.C. (1986). Ap.J., 311, 708 .

Lattanzio, J.C. (1987a). In Late Stages of Stellar Evolution, eds. S.

Kwok \& S.R. Pottasch, p. 235. Dordrecht: Reidel.

Lattanzio, J. C. (1987b) . Ap.J.,313, L15.

Lattanzio, J. C. (1988a). In Evolution of Peculiar Red Giant Stars, in press.

Lattanzio, J. C. (1988b). In Origin and Distribution of the Elements, ed. G.J. Mathews, p. 398. Singapore: World Scientific.

Lauterborn, D., Refsdal, S. \& Weigert, A. (1971). Astron. \& Ast rophys. 10,97 .

Lauterborn, D. \& Siquig, R.A. (1974). Ap.J.,191, 589.

Luck, R.E. \& Lambert, D.L. (1981). Ap.J.,245, 1018 .

Maeder, A. \& Mermilliod, J.C. (1981). Astron. \& Astrophys., 93, 136.

Magee, N.H., Mertz, A.T. \& Huebner, W.T. (1984). Ap.J., 283, 264.

Mateo, M. \& Hodge, P. (1986). Ap.J.Suppl.,60, 893.

Matraka, B., Wassermann, C. \& Weigert, A. (1982). Astron.\& Ast rophys.,107, 283.

Meyer-Hofmeister, E. (1972). Astron. \& Astrophys.,16, 282.

Meylan, G. (1987). Astron. \& Astrophys., 184, 144.

Meylan, G, (1988). Astron. \& Astrophys., $\overline{191}, 215$.

Meylan, G. \& Djorgovski, S. (1987). Ap.J.,322, L94.

Nasi, E. Forieri, C. (1988). Astron. \& Astrophys., submitted.

Pel, J. W. (1985). In Cepheids: Theory and Observations, ed. B.F. Madore, p. 1. Cambridge: Cambridge University Press.

Pel, J.W. \& Lub, J. (1978). In The HR Diagram, eds. A.G.D. Philip \& D.S. Hayes, p. 229. Dordrecht: Reidel. 
Persson, S.E., Aaronson, M., Cohen, J.G., Frogel, J.A. \& Matthews, K. (1983). Ap.J., 266, 105.

Reimers, D. (1975). Mem.Soc.Roy.Sci. Liege, 6 serie, tome 8,p. 369.

Renzini, A. (1984a). In Observational Tests of the Stellar Evolution Theory, eds. A. Maeder \& A. Renzini, p. 21. Dordrecht: Reidel.

Renzini, A. (1984b). private communication.

Renzini, A. Voli, M. (1981). Astron. \& Astrophys.,94, 175.

Robertson, J.W. (1972). Ap.J.,177, 473.

Sandage, A. \& Tammann, G.A. (1969). Ap.J.,157, 683.

Searle, L., Sargent, W.L.W. \& Bagnuolo, W.G. (1980). Ap.J.,179, 427.

Serrano, A. (1983). Rev. Mexicana Astron. Astrophys., $\underline{8}, 131$.

Schmidt, E.G. (1984). Ap.J.,285, 501.

Simon, N.R. (1982). Ap.J., 260, L87.

Simon, N.R. (1986). preprint.

Simon, N.R. (1987). In Pulsation and Mass Loss in Stars, eds. R. Stalio \& L.A. Willson, p. 27. Dordrecht: Reidel.

Slettebak, A. (1970). In Stellar Rotation, ed. A. Slettebak p. 3. Dordrecht: Reidel.

Stellingwerf, R.F. (1978). A.J., 83, 1184 .

Stellingwerf, R.F. (1979). Ap.J., $227,935$.

Stift, M.J. (1984). Astron. \& Astrophys.,140, 445.

Storm, J., Andersen, J., Blecha, A. \& Walker, M.F. (1988). Astron. \& Ast rophys. , 190, L18.

Stothers, R. $(1976 \mathrm{a})$. Ap.J., 209, 800.

Stothers, R. (1976b). Ap.J., $\overline{225}, 939$.

Stothers, R. (1981). Ap.J., $3 \overline{29}, 712$.

Stothers, R. \& Chin, C.W. (1977). Ap.J., 211, 189.

Stothers, R. \& Chin, C.W. (1978). Ap.J., $\frac{225}{25}, 939$.

Vandenberg, D.A. \& Bridges, T.J. (1984). Ä. J., 278, 679.

Waldron, W.L. (1984). In The Origin of Non-Radiative Heating/Momentum in Hot Stars, eds. A.B. Underhill \& A.G. Michalitsianos, p. 2358. Washington, D.C.: NASA.

Walker, A.R. (1987). M.N.R.A.S.,225, 627.

Welch, D.L., McLaren, R.A., Madore, B.F. \& McAlary, C.W. (1987). Ap.J., 321, 162 .

Willson, L.A. (1987). In Pulsation and Mass Loss in Stars, eds. R. Stalio \& L. $\bar{A}$. Willson, p. 285. Dordrecht: Reidel.

Willson, L.A. \& Bowen, G.H. (1984). Nature, 312, 429. 